\title{
SUAS: A Novel Soft Underwater Artificial Skin with Capacitive Transducers and Hyperelastic Membrane
}

\author{
Giovanni Gerardo Muscolo $\dagger^{*} \odot$, Giacomo Moretti \\ and Giorgio Cannata
}

\author{
$\dagger$ DIMEAS, Politecnico di Torino, Corso Duca degli Abruzzi, 24, 10129 Torino, Italy \\ $\ddagger$ TeCIP Institute, Scuola Superiore Sant’Anna, Via Moruzzi 1, 56127 Pisa, Italy \\ E-mail: g.moretti@santannapisa.it \\ 【DIBRIS, Università degli Studi di Genova, Via All'Opera Pia 13A, 16145 Genova, Italy \\ E-mail: giorgio.cannata@unige.it
}

(Accepted November 12, 2018. First published online: December 20, 2018)

\begin{abstract}
SUMMARY
The paper presents physical modeling, design, simulations, and experimentation on a novel Soft Underwater Artificial Skin (SUAS) used as tactile sensor. The SUAS functions as an electrostatic capacitive sensor, and it is composed of a hyperelastic membrane used as external cover and oil inside it used to compensate the marine pressure. Simulation has been performed studying and modeling the behavior of the external interface of the SUAS in contact with external concentrated loads in marine environment. Experiments on the external and internal components of the SUAS have been done using two different conductive layers in oil. A first prototype has been realized using a 3D printer. The results of the paper underline how the soft materials permit better adhesion of the conductive layer to the transducers of the SUAS obtaining higher capacitance. The results here presented confirmed the first hypotheses presented in a last work and opened new ways in the large-scale underwater tactile sensor design and development. The investigations are performed in collaboration with a national Italian project named MARIS, regarding the possible extension to the underwater field of the technologies developed within the European project ROBOSKIN.
\end{abstract}

KEYWORDS: Soft underwater tactile sensors; Underwater sensors; Soft underwater robots; Soft Underwater Artificial Skin; Soft membranes; Underwater manipulator; Autonomous Underwater Vehicles (AUVs); Remotely Operated Vehicles (ROVs).

\section{Introduction}

Researchers from the University of Tokyo estimated 80-100 billion metric tons of rare-earth deposits lie in the Pacific Ocean. Europe is also investigating the feasibility of mining the seabed and a European Union-supported project termed "Blue Mining" commenced in 2014. With a duration of 48 months, a budget of $€ 15$ million, and involving 19 European industries and research organizations, the aim is to examine the business case and technological challenges associated with deep-sea mining. ${ }^{1}$

In this context, underwater artificial skin should be explored with the final aim of obtaining novel systems which could be used to recognize obstacles not only during operations and maintenance for offshore oil and gas industry, but also during maritime search and rescue, oceanographic research, underwater archaeology, and environmental monitoring. ${ }^{2}$

* Corresponding author. E-mail: giovanni.muscolo@polito.it 
Underwater tactile sensors are available in literature, but only few works have been found on underwater tactile sensing technologies enabling large-scale experimental activities.

Bemfica et al. ${ }^{3}$ propose an embedded tactile sensor based on optoelectronic components for fingers of an underwater gripper, but the tactile sensor operates in a small area of the underwater gripper (i.e., fingertips). Ding-zhong et al. ${ }^{4}$ propose an optical fiber slide tactile sensor based on the inner modulation mechanism of optical fibers. Its static and dynamic characteristics are analyzed theoretically and simulated in the paper. However, one problem to use optical fibers is underlined by the dynamic control of the system. Lane et al. ${ }^{5}$ propose contact force and slip sensors included within each fingertip of a dexterous hand developed in a European project named AMADEUS and published in 1999. Solutions of this project are of interest for applications in a deep-sea environment, but novel technological solutions have been developed in the tactile sensor field from 1999 until now. In ref. [6], a modular, reconfigurable, and waterproof snake robot for underwater locomotion is presented. The robot uses a custom-designed force/torque sensor inside each joint module, and an experiment which verifies that the joint module is waterproof at a water depth of $5 \mathrm{~m}$ is performed. In general, the resolution of the strain-gauges used in the robot for underwater applications is not a very good choice and could be optimized using other types of sensors (e.g., conductive or optoelectronic sensors). In ref. [7], tactile sensing for underwater operation system based on multi-finger sensors information fusion is presented, but not real deep-sea experiments are performed and also in this case strain-gauges are used. Authors in ref. [8] show that deep-sea environmental requirements for the design of the actuator are the ability to withstand ambient pressure, the robustness against mechanical overload, and the ease of maintenance. The concept for the pressure resistance is based on the idea to reduce the surface of the uncompensated volume by being as tiny as possible and protect just the components that are sensitive to compression. Therefore, all mechanical and most of the electrical components are exposed to ambient pressure and are just separated from the deep-sea environment by a flexible housing. Palli et al. ${ }^{9}$ report the development of a 6 -axis F/T sensor based on optical reflection. The work reported is based on a previous investigation of the basic concepts and implementation tests for an optical 6-axis F/T sensor. ${ }^{3}$ Stuart et al. ${ }^{10}$ demonstrate that suction flow helps by increasing the static coefficient of friction and normal force for both smooth and textured surfaces. The pinching failure prediction using experimental friction estimates adequately describes the general trends of pinching success with and without suction in water. Stuart et al. ${ }^{11}$ proposed underactuated, compliant, tendon-driven robotic hands suited for deep-sea exploration. Aggarwal et al., in ref. [12], developed a deep-sea-capable tactile sensing system for underwater gripper. The results presented in the paper can be considered as a step toward achieving autonomy in underwater and deep-sea environments.

The works presented above show small area tactile sensors (e.g., fingertips) which explored for a long time in literature and for not-waterproof applications (e.g., Oddo et al., ${ }^{13}$ Silvera-Tawil et al., ${ }^{14}$ etc.). On the contrary, a first step toward a waterproof artificial skin can be found in Bruno and Cannata. ${ }^{15}$ Asadnia et al. ${ }^{16}$ show a flexible and surface-mountable array of micro-electromechanical systems artificial hair cell flow sensors. Bandyopadhyay et al ${ }^{17}$ present a modeling of how shark and dolphin skin patterns control transitional wall-turbulence vorticity patterns using spatiotemporal phase reset mechanisms, and Wen et al., in ref. [18], show a biomimetic shark skin with design, fabrication, and hydrodynamic function. Walker and Anderson ${ }^{19}$ investigated the influence of fresh and salt water on dielectric elastomer sensing. The aim of this investigation was to identify issues, perform modifications, and propose a new sensor design suited to wet and underwater applications. Kahn and Tangorra ${ }^{20}$ presented a work with the final aims at understanding the effects of fluidic loading, fin webbing, and target object geometry on strain sensation with robotic fins and beams as robotic fins and beams come into contact with obstacles underwater.

Muscolo and Cannata ${ }^{21}$ present preliminary steps performed to design a large-scale artificial skin for an underwater vehicle manipulator system ${ }^{22}$ (composed of an underwater remotely operated vehicle and a manipulator) underlining physical limits and novel idea to overcome the technical obstacles in an underwater environment (e.g., pressure compensation, corrosion, etc.).

In ref. [23], the authors propose a human-robot interaction algorithm based on an extended Kalman filtering estimation and measurement of physical interaction force through robotic skin. A novel artificial skin based on optoelectronic technology and able to estimate both normal and shear contact force components is proposed in ref. [24]. In ref. [25], the authors present an approach to automatically acquire the kinematic model of a robot equipped with a multi-modal, modular 


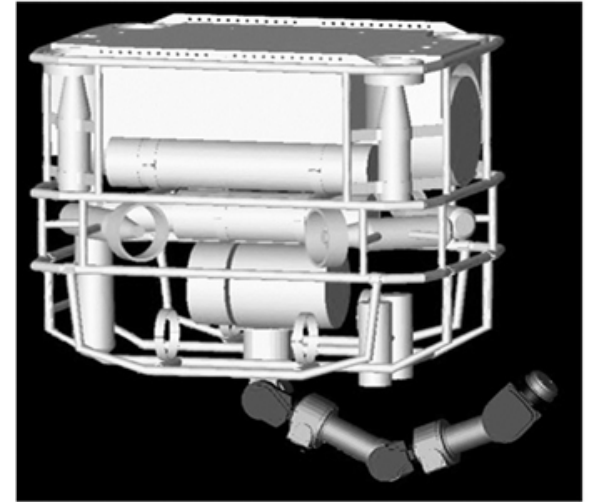

AUV integrated with the underwater robotic arm;

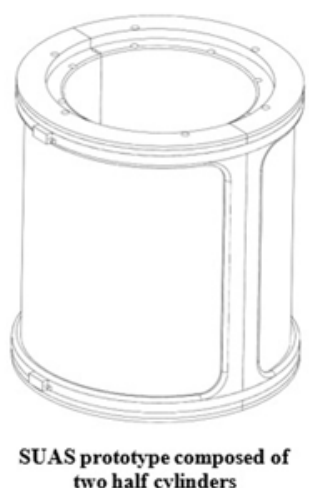

two half cylinders

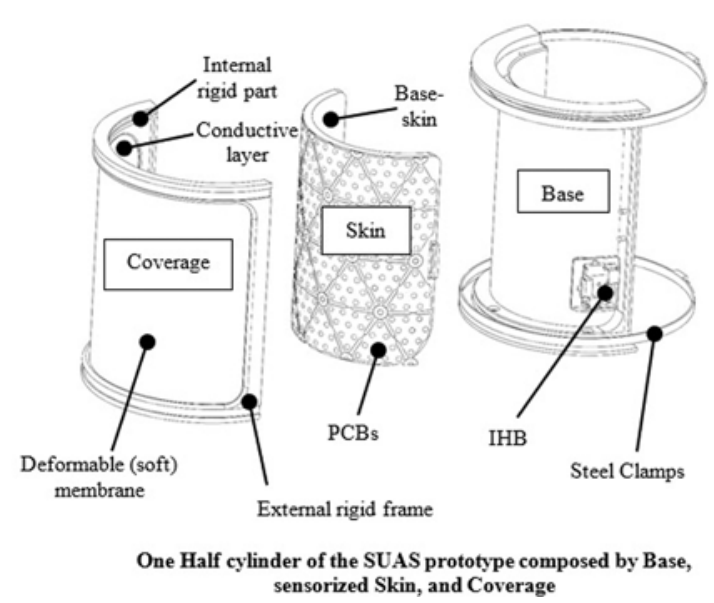

sensorized Skin, and Coverage

Fig. 1. SUAS prototype implemented in an underwater robotic arm.

artificial skin. In ref. [21], the large-scale artificial skin developed for not-waterproof applications within the ROBOSKIN (European research project) ${ }^{26}$ (i.e., to cover the external surface of robots in order to have a more robust human-machine interaction) has been modified and utilized for underwater applications.

In this paper, a Soft Underwater Artificial Skin (SUAS) (see Fig. 1) is proposed, designed, and realized using a 3D printer. SUAS is composed by a compliant polymeric interface (namely, hyperelastic membrane) and a network of electrostatic capacitive sensors (namely, ROBOSKIN artificial skin), capable of sensing the interface deformation. The compliant membrane and the sensing layer are separated by a fluid, aimed at compensating the hydrostatic pressure acting on the skin. Simulations have been performed using hyperplastic membranes in contact with the marine environment and used to cover the ROBOSKIN sensor. ${ }^{26}$ Experimental tests have been done comparing sensor output (SO) signals of tactile sensors modifying external conditions (e.g., sensor in oil, in air, and in contact with different conductive layers). Our proposed SUAS can be used as a distributed force sensor or as a contact detection device.

The design, simulation, and tests of our SUAS prototype are based on results proposed in literature. ${ }^{27-29}$ In particular, in ref. [27], a sensorized robotic finger available in commerce is presented. The objective of the paper is to define the elastomer used to cover the finger composed by a fluid layer. Some tests studied the permeability of the elastomers. It seems that the authors used capacitive transducers immersed in a conductive fluid (water). Five urethanes and four silicones have been tested with standardized procedures and the result is that the silicon Silastic S has a higher value of resistance to abrasion (high strength, low hardness, and high deformation). However, Silastic S absorbs water. Other tests have been performed changing the humidity of the fluid of the finger. The tests underlined that humidity modifies the electromechanical behavior and the conductivity of the fluid. The paper is of interest for our work and helped us to address the experimental tests on 
sensor processing with different fluids and conductive layers. Kampmann et al. ${ }^{28}$ underline how in an underwater environment, integration and miniaturization reduce the effects of mechanical load due to increased ambient pressure up to a point where the components become intrinsically pressure tolerant. From this idea derives the requirement to shrink necessary housing to barely envelop the component and not the whole assembly for an underwater system. Thiede et al. ${ }^{29}$ propose pressuretolerant devices for permanent submerged applications. In particular, to avoid oil compensation, the authors propose soft compounds, like polyurethane or silicon, to transmit the hydrostatic pressure to every housed component and suggest that a pressure-tolerant system with elastic coating is leak-proof and free from any complex filling or refilling procedures unlike liquid compensated systems.

This paper is structured as follows: Section 2 presents artificial skin developed with the ROBOSKIN project and our SUAS prototype; Section 3 presents experimentation on SUAS's capacitive transducers; Section 4 presents physical and analytical modeling of the hyperelastic membrane that covers the SUAS's transducers; Section 5 shows our model and simulation on the fluid-soft membrane interaction; Section 6 shows conclusion and future works.

\section{Soft Underwater Artificial Skin}

\subsection{Technical specifications: underwater manipulation}

Dynamic modeling of an underwater manipulator and its simulation is presented in ref. [30]. The authors in ref. [30] underline the importance to have an artificial skin for underwater applications and have planned future works in this direction using commercial Interlink Force Sensing Resistors to cover its underwater manipulator. The MARIS project ${ }^{31}$ pursues the general objective of studying and further developing the technologies and methodologies enabling the development of underwater robotic intervention systems for their possible applications within underwater scenarios typical for the offshore industry, for the underwater search-and-rescue operations, as well as for the underwater scientific missions. The ROBOSKIN project ${ }^{26,32,33}$ developed a range of new robot capabilities based on the tactile feedback provided by a robotic skin from large areas of the robot body. Within MARIS, the investigations regarding the possible extension to the underwater field of the technologies developed for a terrestrial use within the European project ROBOSKIN ${ }^{26,32,33}$ are currently under development, and this paper will show a symbiotic interaction between the results of the two projects.

MARIS is an acronym used for "MArine Robotics for InterventionS" and it arises as initiative of four Departments of Universities which are members of the Inter-University Center ISME ("Integrated Systems for the Marine Environment"). ${ }^{31}$ The MARIS project is an evolution of the TRIDENT project, ${ }^{34}$ a research program supported by the European Commission and aiming at developing an autonomous system for submarine intervention activities. The general strategic objective of the MARIS project is studying, developing, and integrating technologies and methodologies enabling the development of underwater robotized systems employable for manipulation and transportation activities.

The MARIS system is composed by the Autonomous Underwater Vehicle (AUV) integrated with the robotic arm (see Fig. 1). The vehicle is about $300 \mathrm{~kg}$ and the arm is $30 \mathrm{~kg}$ in air (about $10 \mathrm{~kg}$ in water). Figure 2 shows the 7 DOFs robotic arm: the joint connected with the end effector, the link that connects the shoulder with the wrist where the artificial skin will be attached, and the link attached to the AUV platform presented in ref. [31].

\subsection{ROBOSKIN artificial skin}

The artificial skin developed within the ROBOSKIN project ${ }^{26}$ is used to cover the surface of humanoid robots. An investigation of robot haptic sensing until now has been limited by the lack of tactile sensing technologies enabling large-scale experimental activities, since so far skin technologies and embedded tactile sensors have been mostly demonstrated only at the prototypal stage. The new capabilities pursued with the ROBOSKIN project improved the ability of robots to operate effectively and safely in unconstrained environments and to communicate and cooperate with each other and with humans. The developed artificial skin has been presented in other works showing tactile sensing technology, tactile hardware, software infrastructure, and tactile data processing. ${ }^{26,32,33}$ The transducer consists of a soft dielectric sandwiched by electrodes. ${ }^{26}$ When pressure is applied to 


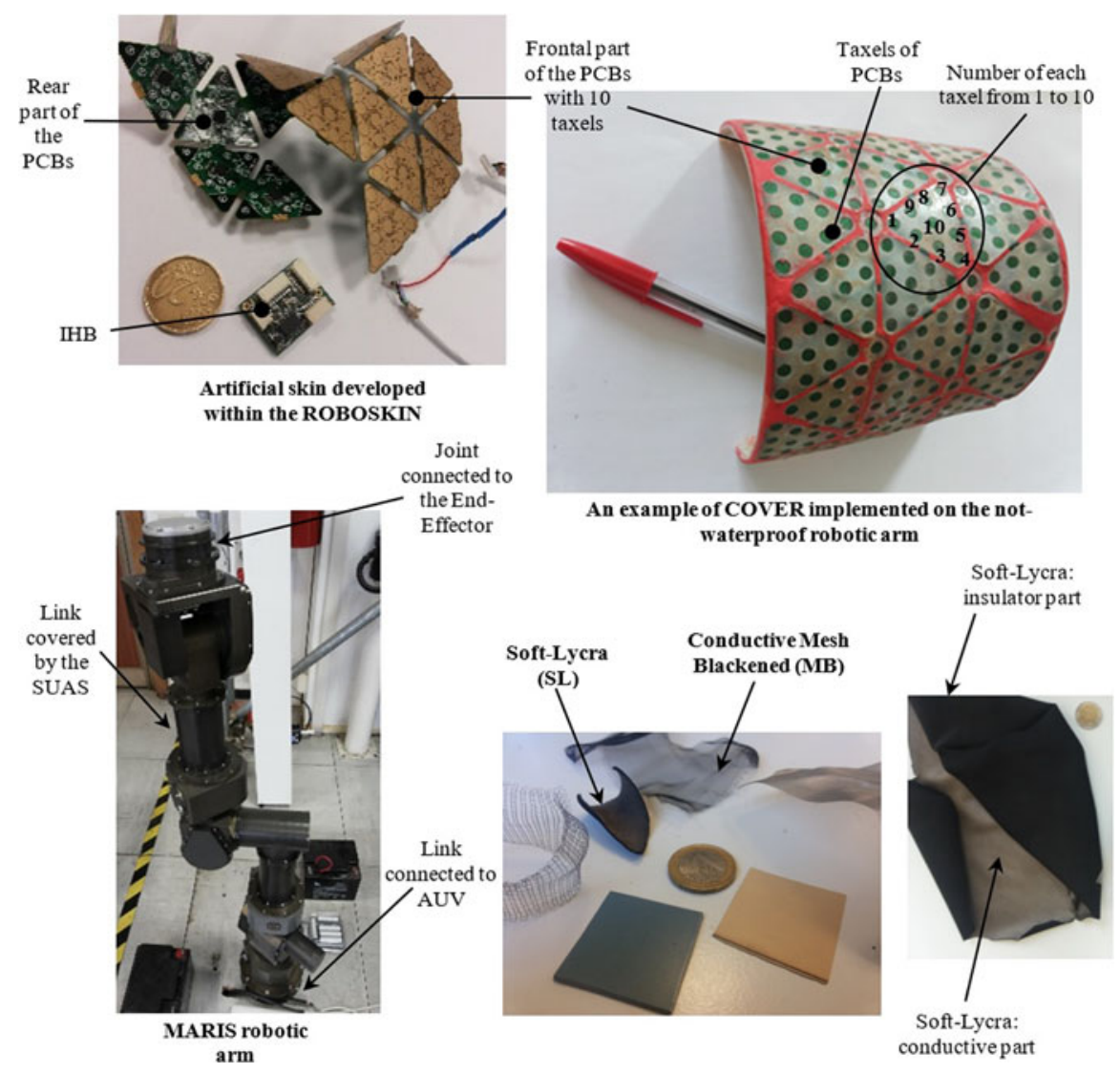

Fig. 2. PCBs composed of 10 taxels and developed with the ROBOSKIN European project. An example of a sensorized cover for robotic arm; 10 taxels are numbered on the cover. The SL and MB are shown. The MARIS 7DOF underwater robotic arm is available at University of Genova and developed by Graal Tech Company.

the sensor, the distance between the electrodes above and below the dielectric changes, and the capacitance changes accordingly. Figure 2 shows the flexible Printed Circuit Board (PCB) including the electronics to obtain 10 measurements of capacitance, one for each taxel. A Capacitance-to-Digital Converter (CDC) (AD7147 from Analog Devices) outputs a digital Sensor Output signal (SO) that allows measurement of the capacitance of all taxels with 16-bit resolution. However, we use only 8-bit measurements for which one measurement unit corresponds to $2.88 \mathrm{fF}$. The shape of the PCB is an equilateral triangle, with edges $24 \mathrm{~mm}$ long. Three communication ports placed along the sides of the triangle relay the signals from one triangle to the adjacent ones. Up to 32 triangles can be connected in this way (4 serial buses with 4 different addresses each), and only one of them needs to be connected to a microcontroller board (IHB) with four connections: one for alimentation; two for connection to PCBs; and one used as interface with computer. This is a critical advantage since it reduces the amount of wires and electrical connections that are required.

Figure 2 shows an example of a sensorized cover developed for a robotic arm. The cover is developed in acrylonitrile butadiene styrene and PCBs shown in Fig. 2 are glued on curved surface. A protective Soft-Lycra (SL) layer is used to cover the PCBs and it is composed of two layers: one internal and conductive in contact with PCBs, and an external one insulating by the external environment.

\subsection{SUAS prototype}

We propose an SUAS able to map the tactile contact of an underwater robotic arm. Figure 3 shows a scheme of the underwater artificial skin which is composed by three elements: (1) a soft membrane; (2) a conductive layer, adherent to the membrane inner face; and (3) capacitive transducers (taxels on the PCB of Fig. 2). Elements (2) and (3) are in oil. Element (1) is used to separate the underwater external environment from the oil inside the system, as presented in ref. [21]. 


\section{Marine Water}

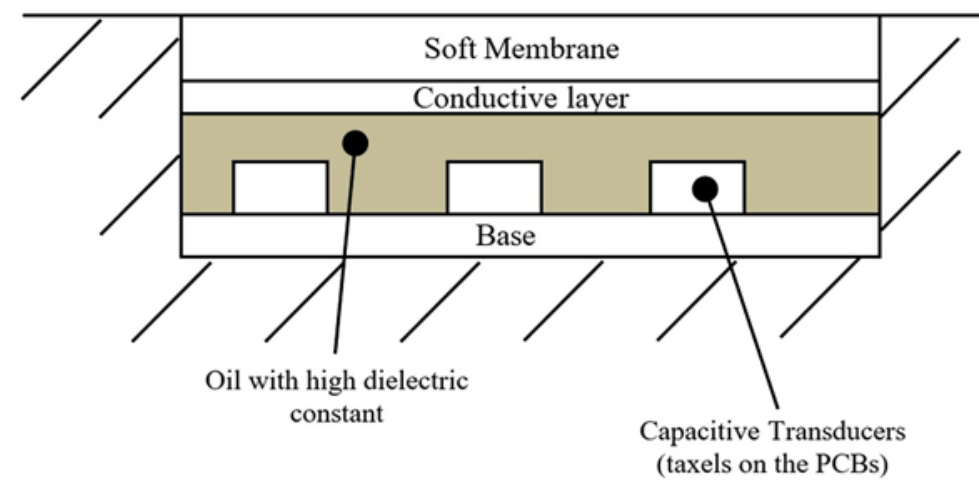

Fig. 3. Oil used as insulator between capacitive transducers (taxels on the PCBs) and conductive layer.

When an object is immersed in marine water, the hydrostatic pressure $\left(p_{m}\right)$ on the object is proportional to the specific weight $\left(\gamma_{s}\right)$ of the fluid (marine water) and to the distance $(h)$ of the object with respect to the plane of the hydrostatic relative loads, assuming coincident with the surface of the sea. The pressure on the sea surface is $p_{a}=1 \mathrm{~atm}=101.3 \mathrm{KPa}$. Our target goal is to obtain an artificial skin able to work in a maximum depth $h=20 \mathrm{~m}$. For marine water $\gamma_{s}=10.051 \mathrm{KN} / \mathrm{m}^{3}$ and the maximum value of the pressure is $p_{m}=\gamma_{s} h+p_{a}=302.3 \mathrm{KPa}$.

When the artificial skin is immersed in an underwater environment, an equilibrium between internal (oil) and external (marine water) pressure is created and it is a function of all involved sub-systems (e.g., rigid materials, soft materials, fluids, electronics, etc.). If an external force is applied at a point on the artificial skin, the equilibrium of the forces is a function of all involved sub-systems and of the intensity of the applied force in the point of contact. If the external force produces a local motion of the membrane, the distance from the conductive layer on the membrane in contact with oil and taxels on the PCBs will be reduced. This leads to a change in capacitance $C=\left(A \varepsilon_{o} \varepsilon_{r}\right) / d$, where $A$ is the overlapping area of the taxel ( $\varepsilon_{o}$ is the vacuum permittivity), $\varepsilon_{r}$ is the relative permittivity of the dielectric material (oil in our case), and $d$ is the distance between taxels and conductive layer.

Figure 1 shows the SUAS prototype attached to the link which connects the shoulder with the wrist of the robotic arm. The link is a cylinder with internal and external diameter, respectively, of 80 and $85 \mathrm{~mm}$, and internal length of $128 \mathrm{~mm}$. Cables and wires transmit information and power supply to all actuators of the robot and are completely in oil in order to compensate the external underwater pressure. The exteroceptive perception of this link will be used to help manipulation and mapping contacts with external environment (i.e., sea, other vehicles, robotics arms, humans, flora and fauna of a deep-sea environment). Figure 1 shows the digital mock-up of the SUAS prototype constituted by two half cylinders fixed together with screws and to the link of the robotic arm. Every half cylinder consists of a base, skin, and coverage:

- The base is designed to be attached to the link of the robotic arm using the same screws to connect the link with shoulder and wrist. In order to stay in an underwater environment for a long time without degradation on its surface, the recommended material used for the SUAS base is Duplex stainless steel. Aluminum anodized (Ergal) is an economic alternative to Duplex.

- The skin consists of three layers: a base-skin in polyvinyl chloride with holes for positioning the CDC of each PCB module constituted by an equilateral triangle; a coverage-skin in epoxy resin (or silicon) to protect PCBs; and a layer with oil to compensate underwater pressure. The skin is fixed to the base using glue and screws.

- The coverage consists of four parts: a first rigid part fixed to the base; a conductive layer; a deformable soft membrane constituted by ethylene propylene diene monomer (M-class) rubber (EPDM) (polyurethane, neoprene, polyethylene, or elastomer membrane); a second rigid part which is fixed to the other rigid part in order to block the edge borders of the soft membrane, allowing deformation only on its internal part. The material used for the rigid parts of the coverage is the same as those of the base.

The total weight of a half cylinder is $0.626 \mathrm{~kg}$ using aluminum anodized (Ergal). 


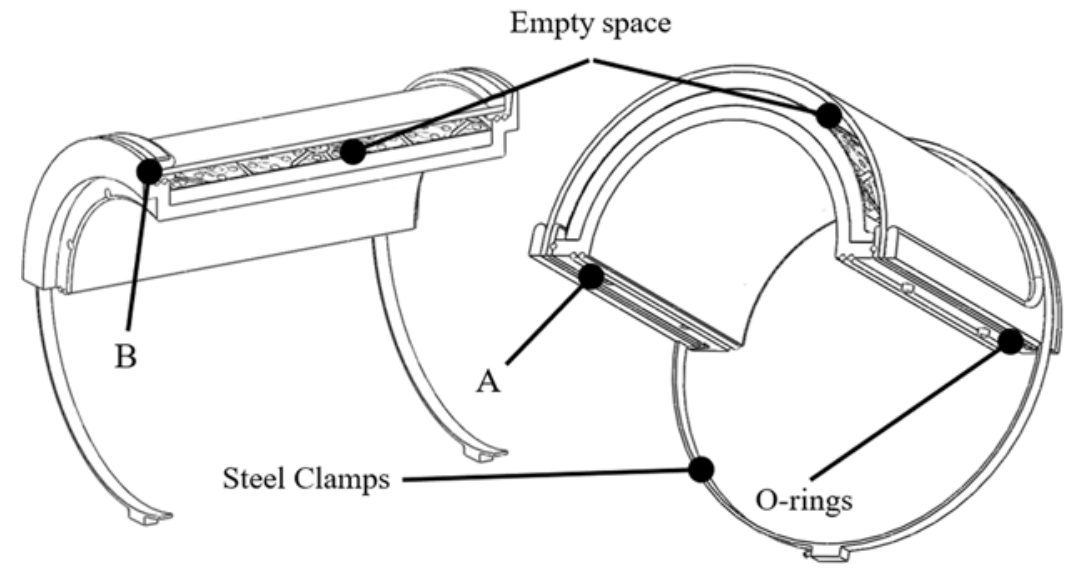

Fig. 4. Frontal and side section of the half cylinder.

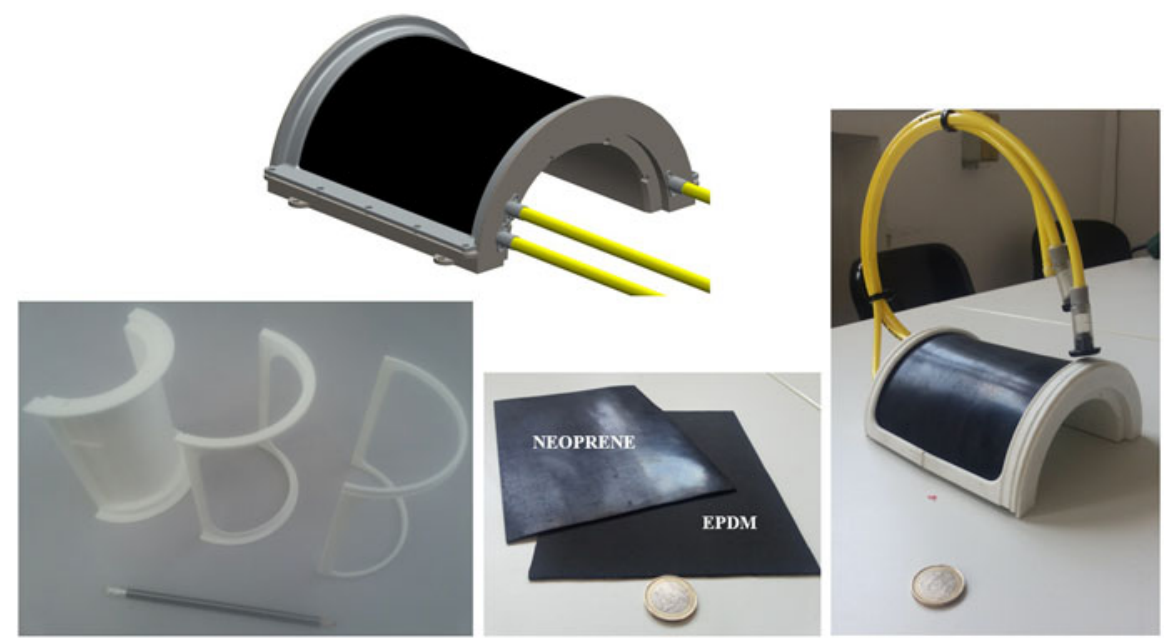

Fig. 5. Digital mock-up of the complete half cylinder of the SUAS prototype; realized parts in PLA; soft membranes used in the prototype (neoprene or EPDM); prototype assembled with the soft membrane (neoprene). [21].

The procedure used to assemble all three parts (base, skin, and coverage) was described in ref.

Figure 4 shows the frontal and side section of the half cylinder presented in Fig. 1. O-rings are used between the two half cylinders (point A) and between the base and the coverage (points A and B). Steel clamps are used to fix the two half cylinders in place of screws in order to reduce the side dimension of the rigid parts.

A first low-cost half cylinder of the SUAS prototype has been realized using a 3D printer. Figure 5 shows the first parts of the SUAS prototype in polyactic acid (or polyactide, PLA) and the final assembly including the soft material. Three tubes are used in each half cylinder: one for PCB's wires and two for oil (in/out). The tube used for wires is filled with silicon resin avoiding inside inclusion of water or oil. The two tubes used for oil are designed with the objective to inlet oil and outlet air inside the closed half cylinder.

The ultimate version of the SUAS prototype is under development in anodized aluminum (Ergal), and it retains all the advantages noted in the three prototype versions: steel clamps of the third prototype version are substituted with screws between two half cylinders and between the base and coverage such as in the second prototype; O-rings between two half cylinders of the third prototype are not used; the coverage consists of a rigid part as in the first prototype; critical points A and B of Fig. 4 have been solved attaching O-rings to the rigid parts of the coverage, simplifying manufacture of the base, and including soft membranes in contact with rigid parts. Figure 6 shows the rear and frontal part of a sheet of triangles as available by supplier and composed by 48 triangles. In order 

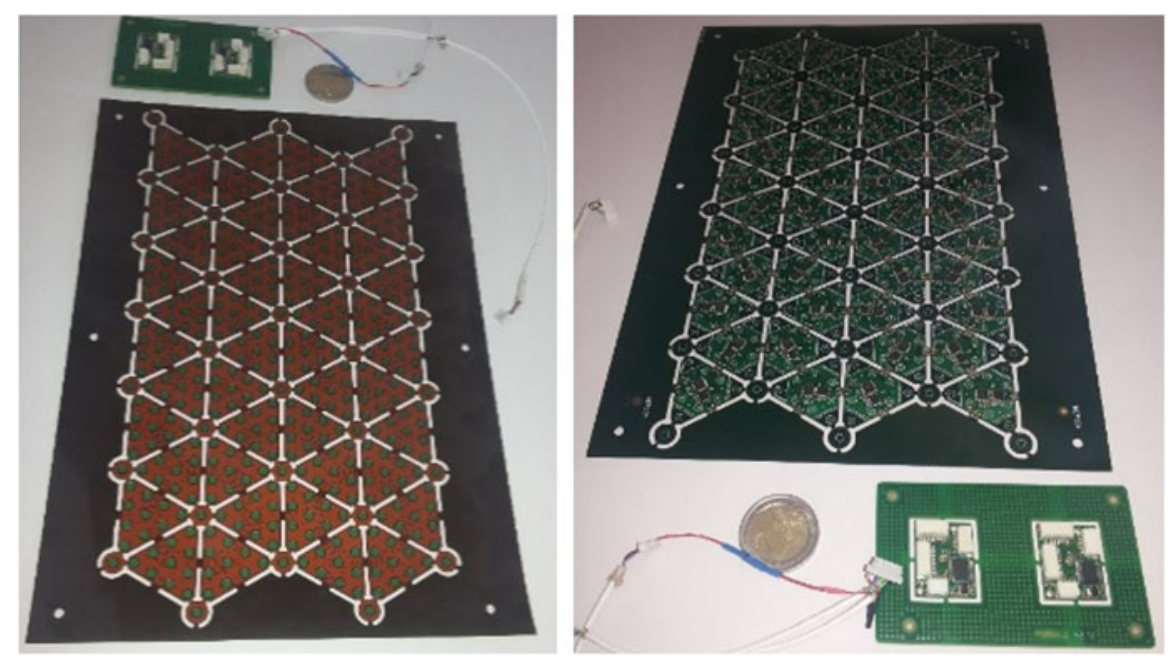

Fig. 6. Frontal and rear part of a sheet of triangles available by supplier.
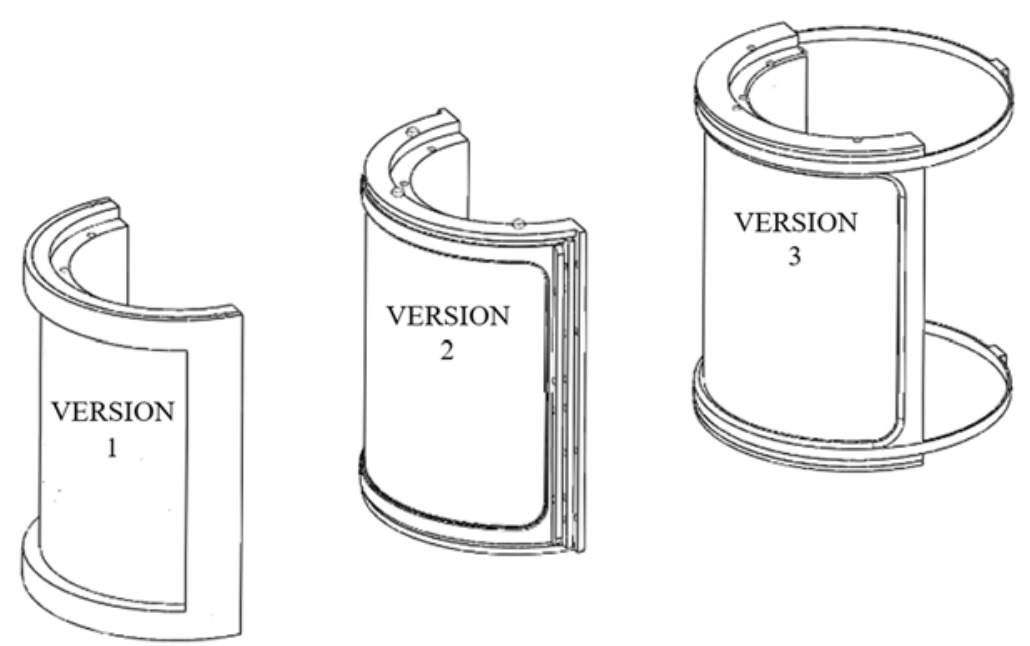

Fig. 7. Comparison among three versions of the half cylinder.

to reduce cables and wires we used directly one sheet of triangles using 32 PCBs adapting it to the curved surface.

\subsection{Design optimization}

In order to optimize the first low-cost prototype, a checkpoint of all designed versions of the half cylinder has been planned with the aim at reducing critical points. In the following, a synthesized comparison of the first three versions is shown (see Fig. 7). Version number 3 of Fig. 7 has been presented and discussed in Figs. 4 and 5; version number 2 has been shown in ref. [21].

In version number 1 (see Fig. 8), the assembly of the two half cylinders is obtained using screws. The coverage of this version is constituted by a rigid part and a soft membrane. Points $\mathrm{C}$ and $\mathrm{D}$, in Fig. 8, show, respectively, how no empty space for oil is available (point C-disadvantage) and how the coverage is fixed to the base with lateral constraints (point D-advantage) avoiding insulation of water. The disadvantage of the point $\mathrm{C}$ could be solved by modifying base and coverage diameters; point B of Fig. 4 is an advantage but if the soft membrane does not have a good adhesion to the base and coverage critical points could be generated. O-rings are positioned between the coverage (constituted by a rigid part) and the base on transversal and longitudinal parts.

The ultimate version under developing in aluminum anodized (Ergal) resumes all the advantages noted in the presented prototype and underlined in the following: steel clamps of version 3 


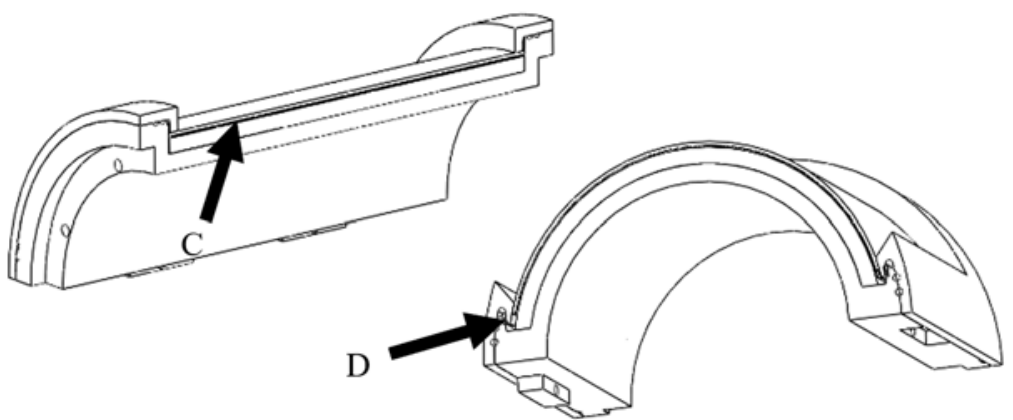

Fig. 8. Frontal and side section of version 1 of the half cylinder.

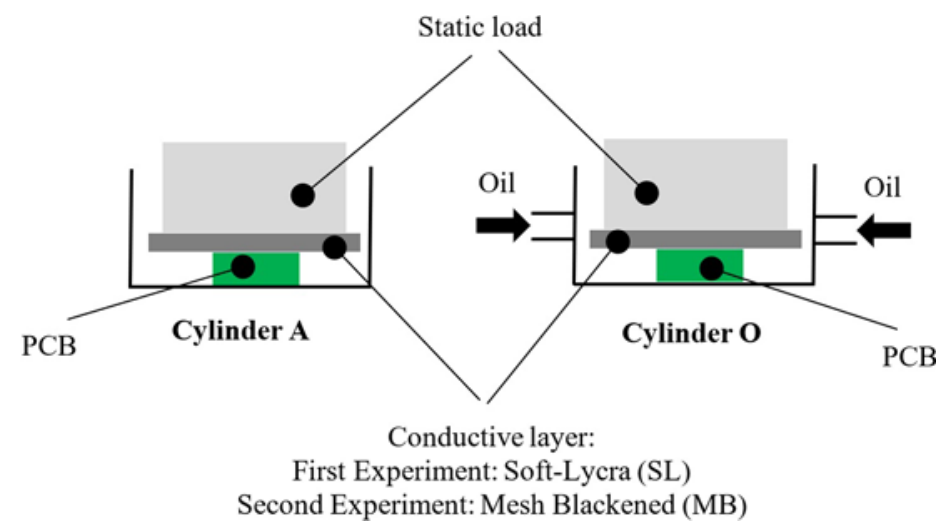

Fig. 9. Sketch of experiments. Left: experiment with PCBs in air; Right: experiments with PCBs in oil.

(Figs. 4 and 5) are substituted with screws between two half cylinders and between base and coverage as in version number 2 (Fig. 7); O-rings between two half cylinders of version 3 are deleted as in versions 1 and 2; the coverage is constituted by a rigid part as in version 1 (Fig. 8); critical points A and B of Fig. 4 have been solved attaching O-rings to the rigid parts of the coverage simplifying manufacture of the base and including soft membranes among contacts of rigid parts; critical points $\mathrm{C}$ and D of Fig. 8 are solved modifying version 1.

\section{Experimentation on Capacitive Transducers}

\subsection{Experimental tests}

In this section, preliminary experiments are presented aimed at investigating the electrostatic interaction among compliant electrode layer, dielectric fluid, and transducer. These experiments are intended to provide indication for the materials selection for the SUAS prototype.

The conductive layer of our SUAS prototype is attached to the soft membrane (as shown in Section 2, Fig. 2) and it works in oil. Figure 9 shows a sketch of the performed experiments. In order to understand the behavior of the PCB in contact with oil and with the conductive layer, the tests were performed forcing the contact of the PCB with the conductive layer in oil and using a static load as shown in Section 5. Two conductive layers were tested in air and oil: the SL obtained attaching the lycra (a commercial product) to a soft tissue, and the conductive Mesh Blackened (MB) (a commercial product). Figure 2 shows some examples of conductive layers, including the two used in our tests. The tests were performed using two PCBs: one in contact with air (in cylinder A, see Figs. 9 and 10) and the other one in contact with oil (in cylinder O, see Figs. 9 and 10). Figure 10 shows cylinder A, cylinder O, the two PCBs, and the two experiments with the two conductive layers (SL and MB). Figure 10(d) shows the static load of $6.5 \mathrm{~N}$ composed by a metal and a layer in polyurethane used as insulator. Figure 10(e) shows cylinder $\mathrm{O}$ with static load and oil inside. Figure 10(f) shows the first experiment performed using two static loads, one for each PCB, and the SL as conductive layer. Figure 10(g) shows the second experiment using MB as conductive layer. In the following, we named PCB_A the PCB in cylinder A and PCB_O the PCB in cylinder $\mathrm{O}$. 
(a)

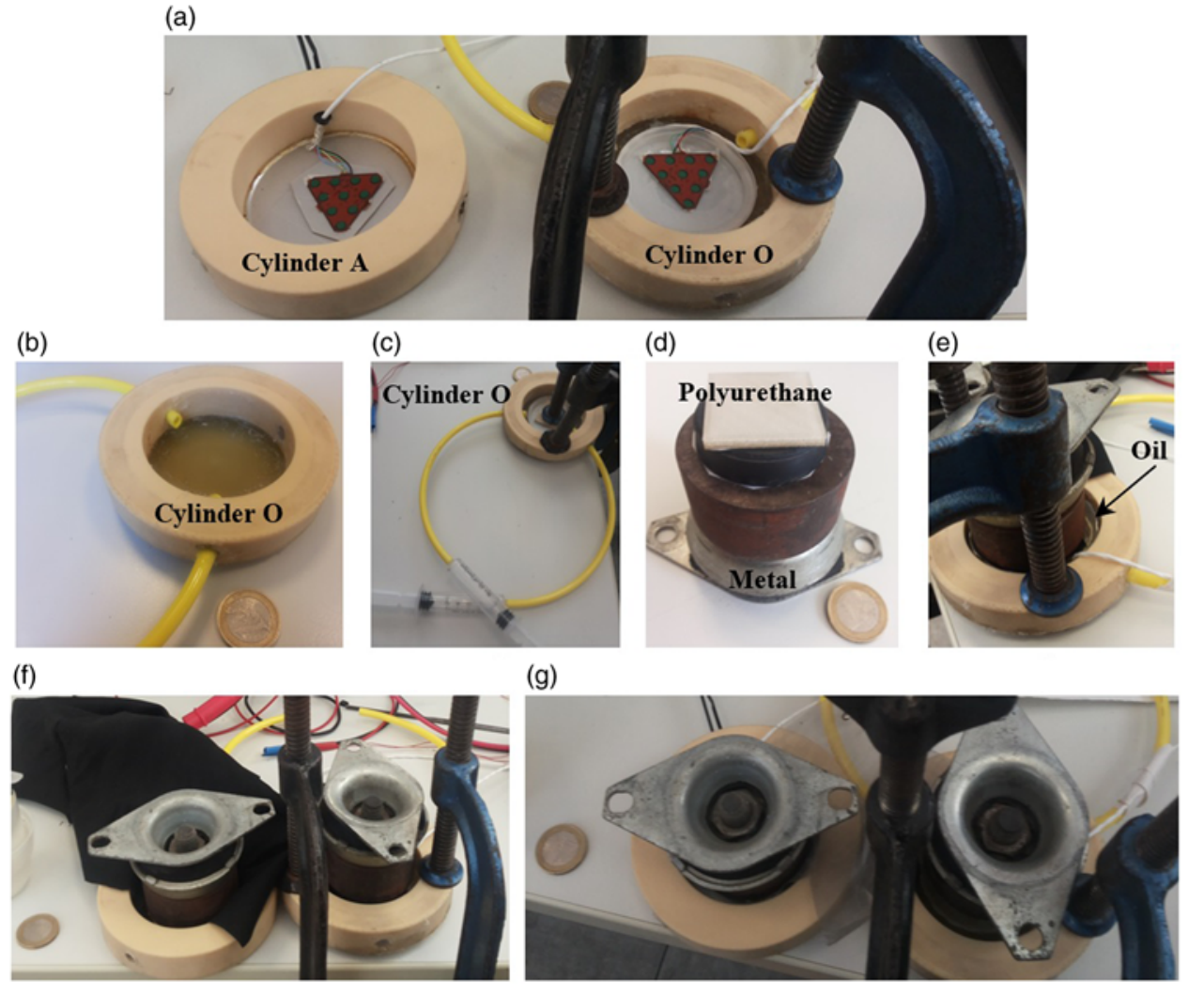

Fig. 10. (a) PCBs in two cylinders for air (cylinder A) and for oil (cylinder O); (b) cylinder for tests with oil (cylinder O); (c) tubes with cylinder $\mathrm{O}$ for tests in oil; (d) static load $(6.5 \mathrm{~N})$ used for tests; (e) oil inside cylinder O; (f) the first experiment using SL as conductive layer; and (g) the second experiment using MB as conductive layer.

The capacitance $(C)$ of the sensors is obtained with

$$
\begin{gathered}
\mathrm{SO}=s+n, \\
C=s \times 3.05 \times 10^{-16},
\end{gathered}
$$

where SO is the sensor digital output signal of each taxel; $s$ is the digital output signal obtained with 16-bit resolution; $n$ is the noise; and $C$ is the capacitance of the transducers measured in Farad. Results are analyzed comparing the SO signals.

\subsection{Results of experiments}

Figure 11 shows a comparison of the SO signals of taxel 10 (the central taxel of the PCB as shown in Fig. 2) before and after including oil in cylinder O using SL as conductive layer. The abscissa shows the time in milliseconds. Results of the first experiment indicate that from 12,000 to $27,000 \mathrm{~ms}$, SO signals increase about from 13,675 to 18,270 after including oil in cylinder O. Figure 12 shows the same comparison of Fig. 11 for each taxel from 1 to 10. The results show that in cylinder O, the difference between the SO signals for each taxel of the PCB before and after including oil is constant.

In the following, for each taxel (from 1 to 10 , see Fig. 2) of each PCB (in cylinder A (PCB_A) and in cylinder O (PCB_O)) a comparison has been done by varying the PCB (PCB_A or PCB_O), the ambient (air or oil), or the conductive layer (SL or MB).

Figures 13-15 show the comparison among the average values of each taxel (1-10 in abscissa) for:

- Two PCBs (PCB_A and PCB_O) using SL and in the same ambient (air) (varying PCB, see Fig. 13).

- The same PCB in cylinder O (PCB_O) using SL or MB and in the same ambient (oil) (varying conductive layer, see Fig. 14). 
(a) Sensor Output signals (SO)

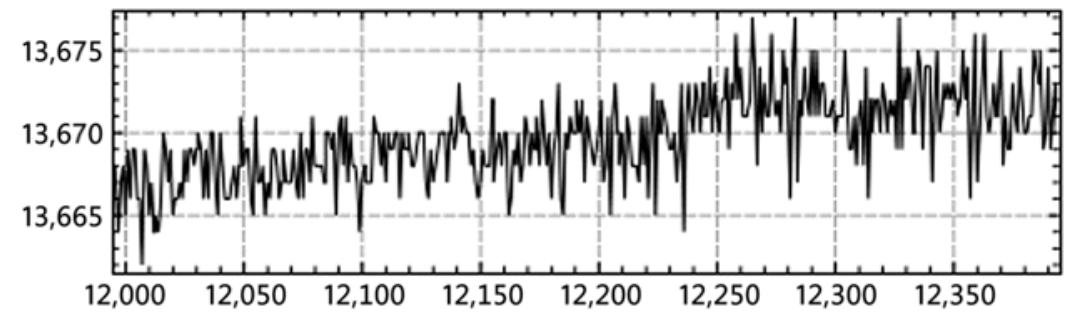

(b)

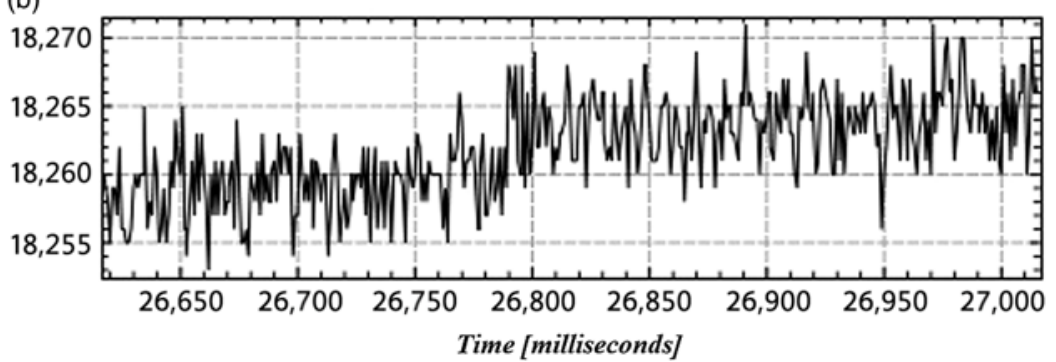

Fig. 11. Comparison of SO signals of taxel 10 of the PCB in cylinder O, during the first experiment and using SL as conductive layer, before including oil (a) and after including oil (b).

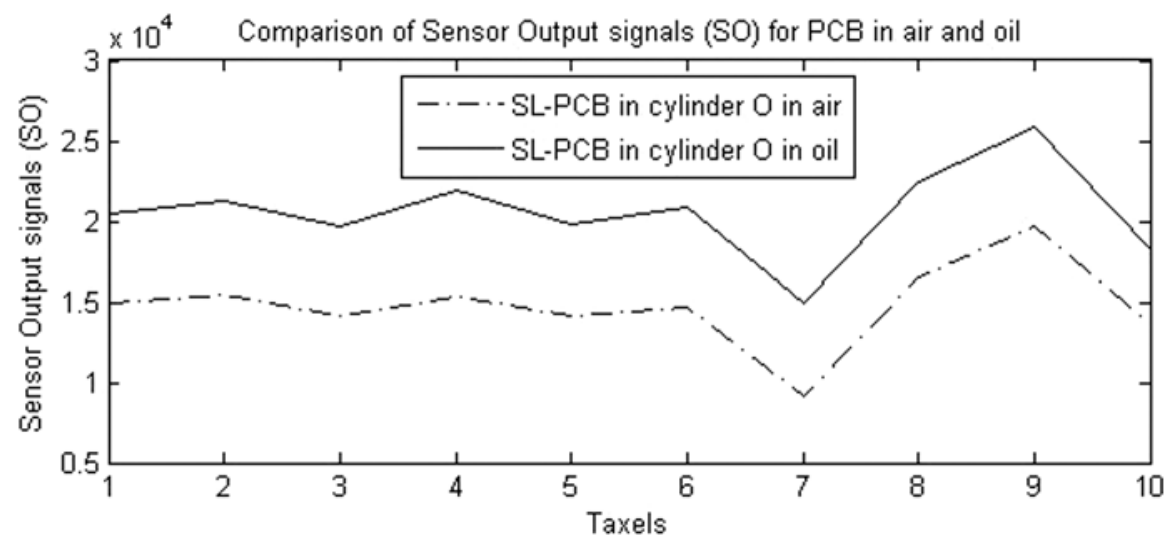

Fig. 12. Comparison of time-averaged SO signals of the 10 taxels (1-10) for the same PCB in cylinder O (PCB_O) in air and oil, using SL as conductive layer.

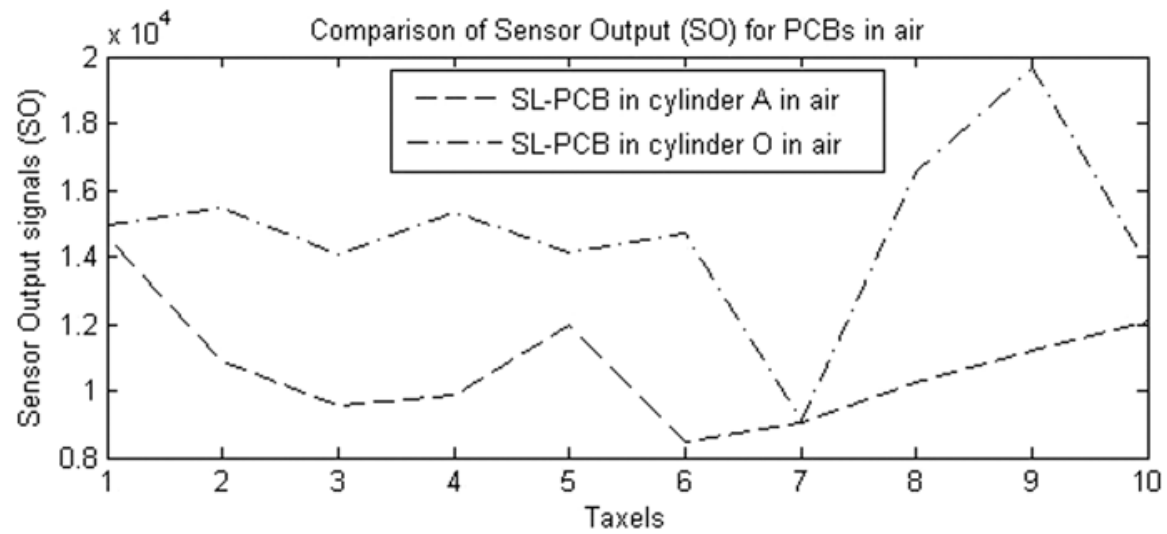

Fig. 13. Comparison of time-averaged SO signals of the 10 taxels (1-10) for the two PCBs in cylinder A (PCB_A) and in cylinder $\mathrm{O}$ (PCB_O) before including oil in cylinder $\mathrm{O}$ and using SL as conductive layer. 


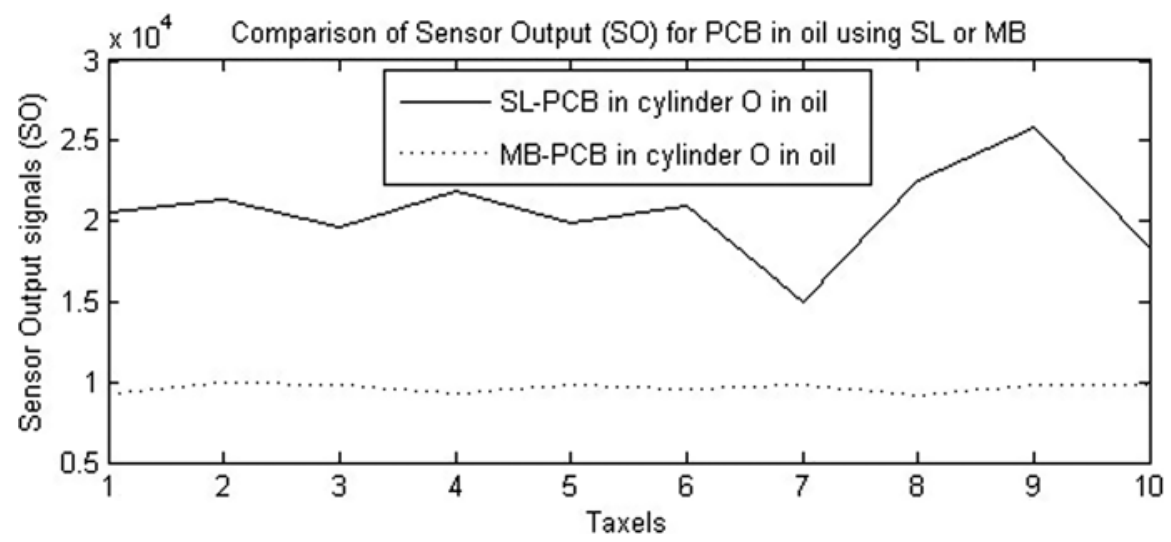

Fig. 14. Comparison of time-averaged SO signals of the 10 taxels (1-10) for the same PCB in cylinder O (PCB_O) in oil and using SL or MB as conductive layers.

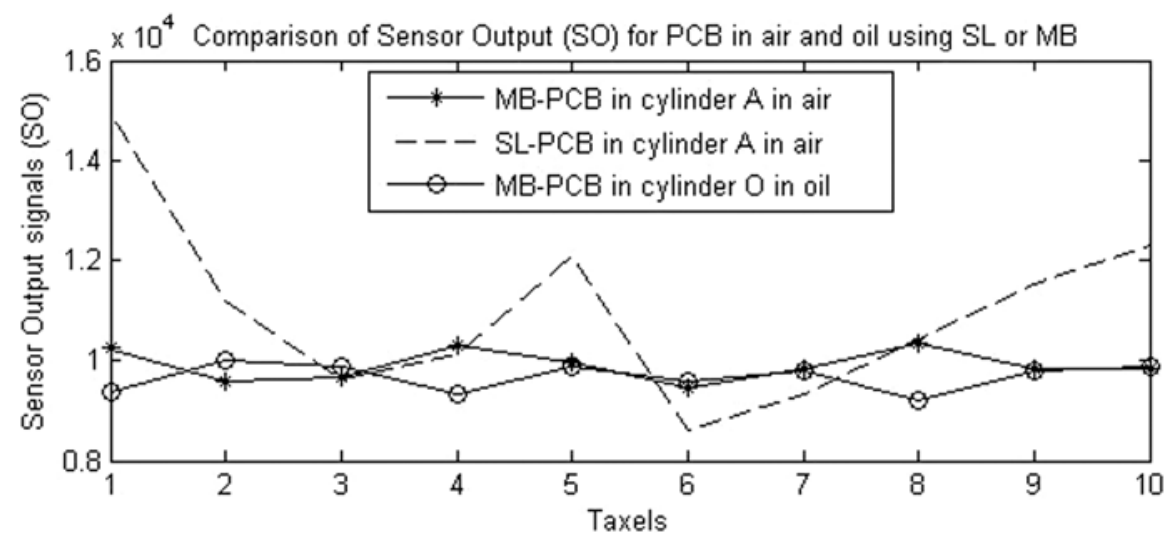

Fig. 15. Comparison of time-averaged SO signals of the 10 taxels (1-10) for the same PCB in cylinder A (PCB_A) and using SL or MB as conductive layers and for the PCB in cylinder O (PCB_O) using MB as conductive layer.

- The same PCB in cylinder A (PCB_A) using SL or MB and in the same ambient (air) (varying conductive layer, see Fig. 15).

- Two PCBs (PCB_A and PCB_O) using MB and in different ambient (air/oil) (varying ambient, see Fig. 15).

\subsection{Discussion}

Results shown in Figs. 13 and 14 indicate that a PCB in oil gives a higher value of capacitance obtained from (2) with respect to the same PCB in air if an SL is used as the conductive layer. In order to confirm this behavior, the second experiment was done using conductive MB in place of SL and results are shown in Fig. 15. In this case, the oil does not give a higher value of capacitance. In order to understand the behavior, we performed comparisons among the PCB_A in air using SL or MB (see Fig. 15). Results of this comparison are shown in Fig. 15 and no significant differences are noted using SL or MB. Figure 14 shows a comparison among each taxel (1-10 in abscissa) of the PCB in oil and using SL or MB. In this case, the same PCB has a different behavior in oil varying the conductive layer.

The results presented above allow us to confirm that low differences are underlined using PCBs in air or oil, but if the conductive layer has a soft interface, the oil provides better adhesion of the conductive layer with taxels giving a higher value of the SO signals and the capacitance using (2). In conclusion, it seems convenient to use lycra with a deformable tissue as used in the presented tests, as it provides potentially higher sensing resolution due to higher capacitance readings. 


\section{Physical and Analytical Modeling of the Hyperelastic Membrane}

\subsection{Deformation gradient}

The deformation of the membrane is considered such as a continuum body occurring when moved from the reference configuration $\Omega_{0}$ to some current configuration $\Omega$. A typical point $X \in \Omega_{0}$ identified by the position vector $\mathbf{X}$ maps into the point $x \in \Omega$ with position vector $\mathbf{x}$. Some researchers deduced the fundamental relation: ${ }^{35}$

$$
\begin{array}{r}
d \mathbf{x}=\mathbf{F}(\mathbf{X}, t) d \mathbf{X}, \\
\mathbf{F}(\mathbf{X}, t)=\operatorname{Grad} \mathbf{x}(\mathbf{X}, t),
\end{array}
$$

where the quantity $\mathbf{F}$ is crucial in nonlinear continuum mechanics and is a primary measure of deformation, called the deformation gradient. In general, $\mathbf{F}$ has nine components for all $t$, and it characterizes the behavior of motion in the neighborhood of a point.

Expression (3) clearly defines a linear transformation which generates a vector $d \mathbf{x}$ by the action of the second-order tensor $\mathbf{F}$ on the vector $d \mathbf{X}$. Hence, Eq. (3) serves as a transformation rule. Therefore, $\mathbf{F}$ is said to be a two-point tensor involving points in two distinct configurations.

Points, curves, tangent vectors, for example, $\mathbf{X}, \Gamma, d \mathbf{X}$, map onto points, curves, tangent vectors $\mathbf{x}, \gamma, d \mathbf{x}$, respectively. An arbitrary differential vector maps via the deformation gradient $\mathbf{F}$ (see the linear transformation (3)). However, a unit vector $\mathbf{N}$ normal to an infinitesimal undeformed surface element $d S$ does not map to a unit vector $\mathbf{n}$ normal to the associated infinitesimal deformed surface element $d s$ via $\mathbf{F}$. Some researchers performed the change in volume between the reference and the current configuration at time $t$.

$$
\begin{array}{r}
d v=J(\mathbf{X}, t) d V, \\
J(\mathbf{X}, t)=\operatorname{det} \mathbf{F}(\mathbf{X}, t)>0,
\end{array}
$$

in which $J$ is the determinant of the deformation gradient $\mathbf{F}$, known as the volume ratio (or Jacobian determinant). In (5), $d V$ and $d v$ denote infinitesimal volume elements defined in the reference and current configurations called undeformed and deformed volume elements, respectively. Further, the volume is assumed as a continuous (or at least a piecewise continuous) function of continuum particles so that $d V=d X_{1} d X_{2} d X_{3}$ and $d v=d x_{1} d x_{2} d x_{3}$ (continuum idealization).

Since $\mathbf{F}$ is invertible we have $J(\mathbf{X}, t)=\operatorname{det} \mathbf{F}(\mathbf{X}, t) \neq 0$. Because of the impenetrability of matter, that is, volume elements cannot have negative volumes, we reject $J(\mathbf{X}, t)<0$ which is mathematically possible. Consequently, the volume ratio $J(\mathbf{X}, t)>0$ must be greater than zero for all $\mathbf{X} \in \Omega_{0}$ and for all time $t$.

If there is no motion $(\mathbf{F}=\mathbf{I}$ and $\mathbf{x}=\mathbf{X})$, we obtain the consistency condition $J=1$, since $\operatorname{det} \mathbf{F}=$ $\operatorname{det} \mathbf{I}=1$. However, a motion (or a deformation) with $J=1$ (at every particle in every configuration and time $t$ ) is called isochoric or volume-preserving. It keeps the volume constant.

The vector elements of the infinitesimally small areas $d \mathbf{s}$ and $d \mathbf{S}$ on the current and reference configurations are related to the Nanson's formula:

$$
d \mathbf{s}=J \mathbf{F}^{-T} d \mathbf{S} .
$$

\subsection{Hyperelastic material}

A so-called hyperelastic material (or in the literature often called a Green-elastic material) postulates the existence of a Helmholtz free-energy function $\Psi$, which is defined per unit reference volume than per unit mass. ${ }^{35-41}$ For the case in which $\Psi=\Psi(\mathbf{F})$ is solely a function of $\mathbf{F}$ or some strain tensor, the Helmholtz free-energy function is referred to as the strain-energy function or stored energy function. We now restrict attention to homogeneous materials in which the distributions of the internal constituents are assumed to be uniform on the continuum scale. For this type of ideal material the strain-energy function $\Psi$ depends only upon the deformation gradient $\mathbf{F}$.

A hyperelastic material is defined as a subclass of an elastic material:

$$
\mathbf{P}=\frac{\partial \Psi(\mathbf{F})}{\partial \mathbf{F}}
$$


And for the symmetric Cauchy stress tensor,

$$
\sigma=J^{-1} \frac{\partial \Psi(\mathbf{F})}{\partial(\mathbf{F})} \mathbf{F}^{T}=J^{-1} \mathbf{F}\left(\frac{\partial \Psi(\mathbf{F})}{\partial(\mathbf{F})}\right)^{T} .
$$

These types of equation are already known as (purely mechanical) constitutive equations (or equations of state). They establish an axiomatic or empirical model as the basis for approximating the behavior of a real material. Such a model the researchers call a material model or a constitutive model. As is clear from the constitutive Eqs. (8) and (9), the stress response of hyperelastic materials are derived from a given scalar-valued energy function, which implies that hyperelasticity has a conservative structure. The derivative of the scalar-valued function $\Psi$ with respect to the tensor variable F determines the gradient of $\Psi$. It is a second-order tensor which we know as the Piola-Kirchhoff tensor $\mathbf{P}$.

\subsection{Extension and compression of a rod}

Considering an extension or compression of a rod (with uniform cross-section) in the direction of the $x_{1}$ axis up to the stretch $\lambda_{1}$. The associated relation $x_{1}=\lambda_{1} X_{1}$ defines a uniform deformation along the $x_{1}$ axis. If two stretches, for example, $\lambda_{1}$ and $\lambda_{2}$, can be chosen arbitrarily and the third stretch $\lambda_{3}$ is determined by the condition $J=\lambda_{1} \lambda_{2} \lambda_{3}=1$ (this type of deformation keeps the volume constant), the associated mode of deformation is often referred to as biaxial (although there are changes of lengths in all three directions). A typical biaxial deformation is characterized in the following form:

$$
x_{1}=\lambda_{1} X_{1} ; x_{2}=\lambda_{2} X_{2} ; x_{3}=\frac{1}{\lambda_{1} \lambda_{2}} X_{3} .
$$

To change the shape of a piece of rubber is very much easier than to change its volume. For the purpose of computational analyses, rubber is often regarded as incompressible with the constraint condition $J=\lambda_{1} \lambda_{2} \lambda_{3}=1$.

A very sophisticated development for simulating incompressible (rubber-like) materials in the phenomenological context is due to Ogden. ${ }^{36,37}$ The postulated strain energy is a function of the principal stretches $\lambda_{a}, a=1,2,3$, which is computationally simple and plays a crucial role in the theory of finite elasticity. It describes the changes of the principal stretches from the reference to the current configuration and has the form

$$
\Psi=\Psi\left(\lambda_{1}, \lambda_{2}, \lambda_{3}\right)=\sum_{p=1}^{N} \frac{\mu_{p}}{\alpha_{p}}\left(\lambda_{1}^{\alpha_{p}}+\lambda_{2}^{\alpha_{p}}+\lambda_{3}^{\alpha_{p}}-3\right) .
$$

In comparison with the linear theory, the (consistency) conditions are obtained:

$$
2 \mu=\sum_{p=1}^{N} \mu_{p} \alpha_{p}, \text { with } \mu_{p} \alpha_{p}>0, \quad p=1, \ldots, N
$$

where the parameter $\mu$ denotes the classical shear modulus in the reference configuration, known from the linear theory. In Eq. (11), $N$ is a positive integer which determines the number of terms in the strain-energy function, $\mu_{p}$ are (constant) shear moduli and $\alpha_{p}$ are dimensionless constants, $p=1, \ldots, N$. It emerges that only three pairs of constants $(N=3)$ are required to give an excellent correlation with experimental stress-deformation data for simple tension, equibiaxial tension, and pure shear of vulcanized rubber over a very large strain range. The typical values of the constants of table determine the shear modulus $\mu=4.225 \times 10^{5} \mathrm{~N} / \mathrm{m}^{2}$ according to (12).

\subsection{Inflation of a spherical balloon}

Inflation experiments of spherical neoprene balloons were carried out by many researchers. In ref. [35], the inflation of a spherical (incompressible rubber) balloon with different material models has been investigated.

We know from a perfect sphere under inflation pressure that every direction in the plane of the sphere is a principal direction. Hence, the stretch ratio is $\lambda=\lambda_{1}=\lambda_{2}$ which characterizes an equibiaxial deformation. The associated circumferential Cauchy stress is $\sigma=\sigma_{1}=\sigma_{2}$ (while 
Table I. Typical values of the constants. ${ }^{35}$

\begin{tabular}{ccr}
\hline $\boldsymbol{a}$ & $\boldsymbol{\alpha}_{\boldsymbol{a}}$ & \multicolumn{1}{c}{$\boldsymbol{\mu}_{\boldsymbol{a}}$} \\
\hline 1 & 1.3 & $6.3 \times 10^{5}$ \\
2 & 5 & $0.012 \times 10^{5}$ \\
3 & -2.0 & $-0.1 \times 10^{5}$ \\
\hline
\end{tabular}

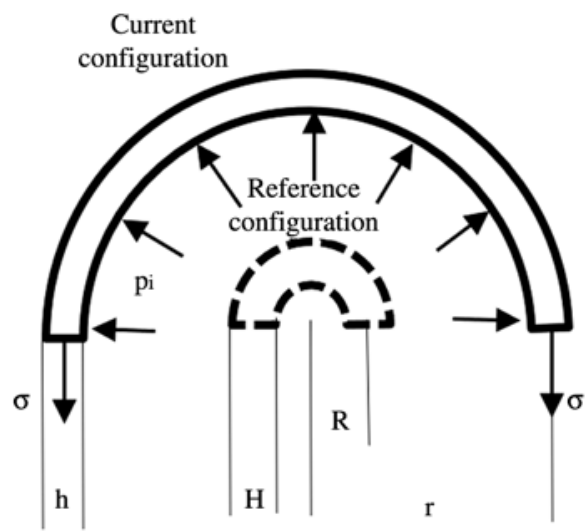

Fig. 16. Geometry of a spherical balloon in the reference and the current configuration, showing only one hemisphere.

$\sigma_{3}=0$ by the assumption of plane stress). Hence, constitutive equations of biaxial deformation reduce to a single relation, namely (see Table I)

$$
\sigma=\sum_{p=1}^{N} \mu_{p}\left(\lambda^{\alpha_{p}}-\lambda^{-2 \alpha_{p}}\right) .
$$

By equilibrium we find from Fig. 16 (free body diagram) that $r^{2} \pi p_{i}=2 r \pi h \sigma$, where $r$ and $h$ denote the radius and the wall thickness of the rubber balloon in the current configuration, respectively. According to this condition, researchers find that

$$
p_{i}=2 \frac{h}{r} \sigma .
$$

In view of the kinematical situation of the inflated balloon (see Fig. 16), the stretch $\lambda$ at a certain point of the balloon is $r / R$. Incompressibility requires that wall volume is conserved which means that $4 \pi r^{2} h=4 \pi R^{2} H$. With this condition researchers find that $\lambda_{3}=h / H=1 / \lambda_{2}$ which denotes the stretch in the direction perpendicular to the surface of the sphere, indeed $\lambda_{1} \lambda_{2} \lambda_{3}=\lambda^{2} \lambda_{3}=1$.

Using these relations and constitutive Eq. (13), researchers find from (14) the analytical expression

$$
p_{i}=2 \frac{H}{R} \sum_{p=1}^{N} \mu_{p}\left(\lambda^{\alpha_{p}-3}-\lambda^{-2 \alpha_{p}-3}\right) .
$$

Vertechy et al ${ }^{41}$ proposed a reduced model and application of inflating circular diaphragm dielectric elastomer generators for wave energy harvesting. In this work, an inflated circular diaphragm dielectric elastomer generator is shown. It consists of an equibiaxially prestretched planar circular dielectric elastomer membrane that is clamped along its perimeter with a radius and a thickness (radius and thickness of the dielectric elastomer membrane in its planar undeformed state are different from the radius and thickness in the deformed state). When the opposing sides of the inflated circular diaphragm dielectric elastomer generator are subjected to a differential pressure, $p$, and to an electric potential difference, $V$, it undergoes an out-of-plane axial-symmetric (bubble-like) deformation (area expansion). 


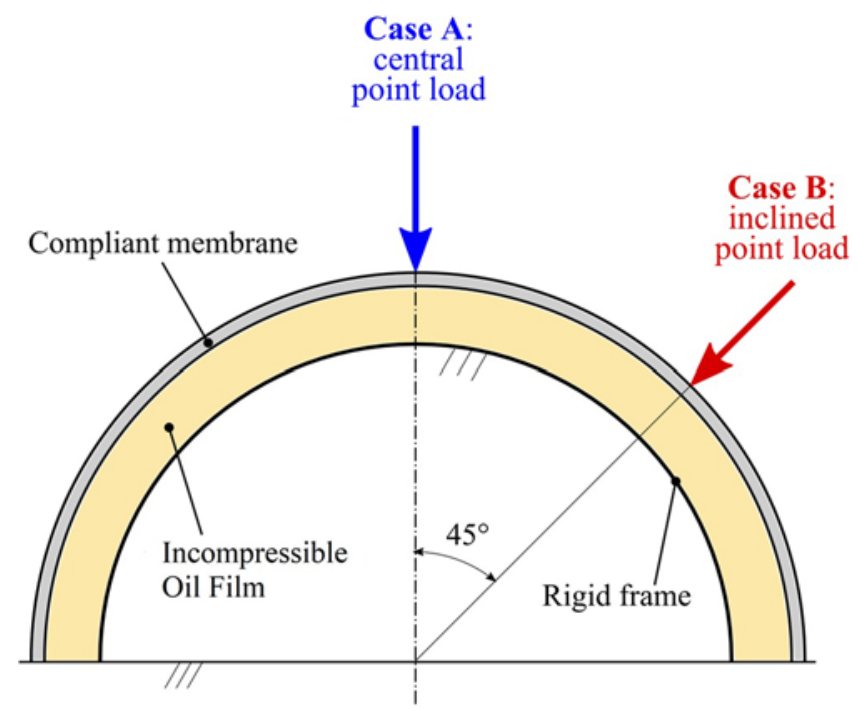

Fig. 17. Schematic of the domain simulated via FE method and of the loading conditions considered.

Dorfmann and Ogden ${ }^{37}$ propose a solution for an extension and inflation of a thick-walled tube which is more related to our application.

\section{Static Model of the Fluid-Soft Membrane Interaction}

In this section, a static Finite Element (FE) model of an SUAS soft membrane subjected to point loads is presented.

A schematic representation of the portion of device simulated via FE method is provided in Fig. 17. The device is treated as bi-dimensional, and it is assumed to be in a plain-strain condition, that is, the strain along the direction perpendicular to the view in Fig. 17 is null. The polymeric membrane is assumed to have hemicylindrical shape and uniform thickness in the undeformed configuration. The membrane subdomain is treated as an incompressible hyperelastic continuum, ${ }^{35}$ subjected to external loads on the external face and to oil pressure on the inner face. The conductive layer on the membrane inner face has not been kept into account, as its stiffness is assumed negligible (e.g., it is much thinner than the membrane).

In the presented simulations, oil response is emulated by means of a uniform pressure (as the system is considered static) and the total oil volume contacting the membrane is assumed to be constant owing to incompressibility. The proposed modeling approach applies as long as the membrane does not come into direct contact with the rigid inner wall of the oil chamber (housing the taxels of the SUAS).

Since the elastomeric membrane thickness is small compared to its curvature, the membrane is treated as a thin shell, and, being incompressible, it results in equilibrium (in the undeformed configuration) under the effect of two equal pressures applied on opposite faces (i.e., sea water pressure on the outer face and oil pressure inside) regardless of their absolute value. For the same reason, the effect of gravity-induced fluid pressure differential on the membrane response is neglected, as, at the equilibrium, the pressure gradient within the inner and outer fluid is the same.

Attention is entirely set on the determination of the oil pressure variation due to the external loads. Two loading scenarios are examined (see Fig. 17): (A) a central load (i.e., force per unit device width) is applied on the lateral surface of the membrane, in correspondence with the transversal symmetry axis of the device; (B) the same load is applied on the membrane lateral surface, with an inclination of $45^{\circ}$ with respect to the transversal symmetry axis.

\subsection{Hyperelastic membrane model}

Rubber-like materials are most often incompressible and present a large-strain mechanical behavior which is described by means of nonlinear hyperelastic models. ${ }^{35}$ In hyperelastic materials, stress and deformation can be related by means of a strain-energy function, $\Psi$, that provides the local elastic energy density stored in the material as a function of the deformation. Previous works have shown 
that polymers like those used to build the soft membrane in SUAS can be treated as incompressible hyperelastic materials. ${ }^{36-40}$

Using well-established formalisms of continuum mechanics, the deformation of the membrane is described by a "deformation function", $\chi$, which maps each point of the continuum, $\mathbf{X}$, in the reference undeformed configuration to a corresponding point, $\mathbf{x}$, in the actual deformed configuration:

$$
\mathbf{x}=\chi(\mathrm{X}, t)
$$

The gradient of $\chi$ (namely "deformation gradient") is denoted by $\mathbf{F}=\partial \mathbf{x} / \partial \mathbf{X}$, and it intervenes in the definition of the right Cauchy-Green tensor:

$$
\boldsymbol{C}=\mathbf{F}^{T} \mathbf{F}
$$

where superscript $T$ indicates the transpose operation. Tensor $\boldsymbol{C}$ provides the "principal deformation directions" that coincide with an orthonormal set of eigenvectors of $\boldsymbol{C}$. Besides, the square roots of the eigenvalues of $\boldsymbol{C}$ represent the "stretches along the principal deformation directions" (or "principal stretches") that are indicated with $\lambda_{1}, \lambda_{2}$, and $\lambda_{3}$ and, in hyperelastic continuum mechanics, are used in place of the strain as a metric of the deformation. Physically, the stretch provides a measure of the ratio between the deformed and undeformed length of a material fiber along a prescribed direction.

Owing to incompressibility, the following relationship among the principal stretches holds:

$$
\lambda_{1} \lambda_{2} \lambda_{3}=1
$$

Stress components in the deformed continuum can be computed using the strain-energy function, $\Psi(\mathbf{F})$, that expresses the volumetric energy density of the material. In particular, for an incompressible material, the Cauchy stress tensor can be computed from $\Psi$ as follows:

$$
\sigma=\frac{\partial \Psi(\mathbf{F})}{\partial(\mathbf{F})} \mathbf{F}^{T}-p \mathbf{I}
$$

where $p$ is an indeterminate multiplier which is resolved by prescribing the incompressibility constraint.

Hyperelastic material models seek to provide a relationship for $\Psi$ as a function of $\mathbf{F}$. It can be shown $^{35}$ that, for hyperelastic isotropic materials, $\Psi$ can be expressed as a function solely of the principal invariants of the tensor $\boldsymbol{C}$ :

$$
\begin{aligned}
\Psi & =\Psi\left(I_{1}, I_{2}, I_{3}\right) \\
I_{1}=\lambda_{1}^{2}+\lambda_{2}^{2}+\lambda_{3}^{2} ; I_{2} & =\lambda_{1}^{2} \lambda_{2}^{2}+\lambda_{1}^{2} \lambda_{3}^{2}+\lambda_{2}^{2} \lambda_{3}^{2} ; I_{3}=\lambda_{1}^{2} \lambda_{2}^{2} \lambda_{3}^{2}
\end{aligned}
$$

Constitutive equations for rubber-like materials usually have the general form given by Eq. (20), with the dependence on $I_{3}$ omitted as $I_{3}=1$ for incompressible media (see Eq. (18)). In the specific case under investigation, due to the plain-strain assumption, the out-of-plane direction is a principal deformation direction (for convenience, we indicate it as the third principal deformation direction) and the corresponding stretch in that direction is uniformly unitary: $\lambda_{3}=1$.

Neglecting the mass forces acting on the membrane (e.g., membrane weight), the local static equilibrium condition for the continuum results in the following well-known equation: ${ }^{35}$

$$
\operatorname{div}(\sigma)=0
$$

where the operator $\operatorname{div}(\cdot)$ is the divergence of a tensor.

Equations (19) and (21) combined with a constitutive equation in the general form of Eq. (20) and with proper boundary conditions represent a set of partial differential equations that can be solved via FE method, thus providing a local solution for the membrane stress and stretch states.

Although the FE solver used in this paper solves the described set of continuum equations locally (i.e., everywhere throughout the elastomer domain), a lumped-parameter approach could have been attempted based on the observation that the membrane thickness is small compared to the other dimensions. In ref. [41], for example, the authors study the deformation of an axisymmetric inflated membrane, treating the elastomer as a thin incompressible shell (whose stresses do not vary throughout the membrane thickness), thus obtaining a set of simplified differential equations relating the deformation function components to the stresses. 


\subsection{Fluid-soft membrane interaction}

The fluid-soft membrane interaction is not simple to analyze and simulate, and recent studies are oriented to define novel systems composed of the fluid inside a customized soft structure. The results presented in ref. [42] provided guidance to define the limits of using certain soft materials with respect to others. By analysis of the presented literature, silicon rubber has some good features for the system under investigation, but it provides a membrane with very low stiffness. Fluid Elastomer Actuators (FEAs) can be designed for a pressure range of 20-55 kPa; Pneumatic Artificial Muscles (PAMs) can be designed for a pressure range of 345-689 kPa; and fiber-reinforced FEAs have a pressure range of $172-241 \mathrm{kPa}$. The pressure range considered in this paper (see Section 2) suggests the use of PAMs or soft membranes made of the same material used for PAMs. In particular, neoprene rubber, EPDM (M-class rubber), or polyurethane are here considered as soft materials for the membrane. The three elastomers have applications in a deep-sea environment. Neoprene is the vulcanized rubber used to develop marine suits. ${ }^{43}$ Polyurethane is used in underwater applications, as shown, for example, in ref. [29]. EPDM rubber has a wide range of applications, but it exhibits poor compatibility with most gasoline, kerosene, aromatic, and aliphatic hydrocarbons, halogenated solvents, and concentrated acids, thus its application is possible only upon verification of its chemical compatibility with the oil employed in the SUAS system.

In ref. [44], some studies on materials used in deep-sea environment have been conducted. No visible deterioration caused by marine organisms was detected on the recovered semi rigid polyethylene and polyurethane foams.

\subsection{Case study and results}

In the presented case study, we make reference to EPDM as reference elastomeric material for the membrane.

For EPDM, ref. [40] suggests that the following constitutive equation (in the general form postulated by Eq. (20)) can be assumed:

$$
\Psi=C_{1}\left(I_{1}-3\right)+C_{2}\left(I_{2}-3\right)+C_{3}\left(I_{2}-3\right)^{2}
$$

with the following values for the constitutive parameters: $C_{1}=20.7 \mathrm{MPa}, C_{2}=-4.4 \mathrm{MPa}, C_{3}=$ $77 \mathrm{kPa}$.

The continuum hyperelastic domain is a hemicylindrical shell with an inner radius of $57.5 \mathrm{~mm}$ and a constant thickness of $1.5 \mathrm{~mm}$ in the undeformed configuration. The oil chamber has thickness (in radial direction) of $5 \mathrm{~mm}$. The transducer has total width of $123 \mathrm{~mm}$. For simplicity, it is assumed that the volume of oil housed under the membrane coating is constant, that is, fluid cannot enter/exit through the connected pipes during operation.

Only the membrane subdomain is directly simulated by the FE solver, using a discretization scheme and the above-mentioned equations, while the oil domain is emulated through a uniform pressure and by prescribing the condition that the area subtended by inner face of the compliant membrane is constant (i.e., the fluid film is incompressible).

For boundary conditions, it has been assumed that the inner membrane surface is subjected to the uniform oil pressure difference (with respect to the equilibrium pressure, that equals the external water pressure, and is thus not considered); the outer face is free and is punctually subjected to the prescribed external loads; the membrane lower transversal edges are secured to the frame and are thus fixed.

Simulations are carried out using the Structural Mechanics module of a commercial FE software. The standard set of equations provided by the software has been modified to include non-default constitutive Eq. (23). A structured mesh with 2400 rectangular elements featuring Lagrange shape functions with order two is used. An UMFPACK solver has been selected for the discretized set of equations.

In order to meet the fluid volume conservation condition, the solution (relative to a specific loading condition) is found iteratively: for a given value of oil pressure differential, simulations are run for a set of values of the external load, and the resulting oil volume subtended by the membrane is calculated for each case. The value of the external load that provides equilibrium at the chosen oil pressure is such that in correspondence to which the subtended oil volume equals the initial volume. 
(a)

Case A: central load (oil diff. press.: $10 \mathrm{kPa}$, load: $24.4 \mathrm{~N}$ )
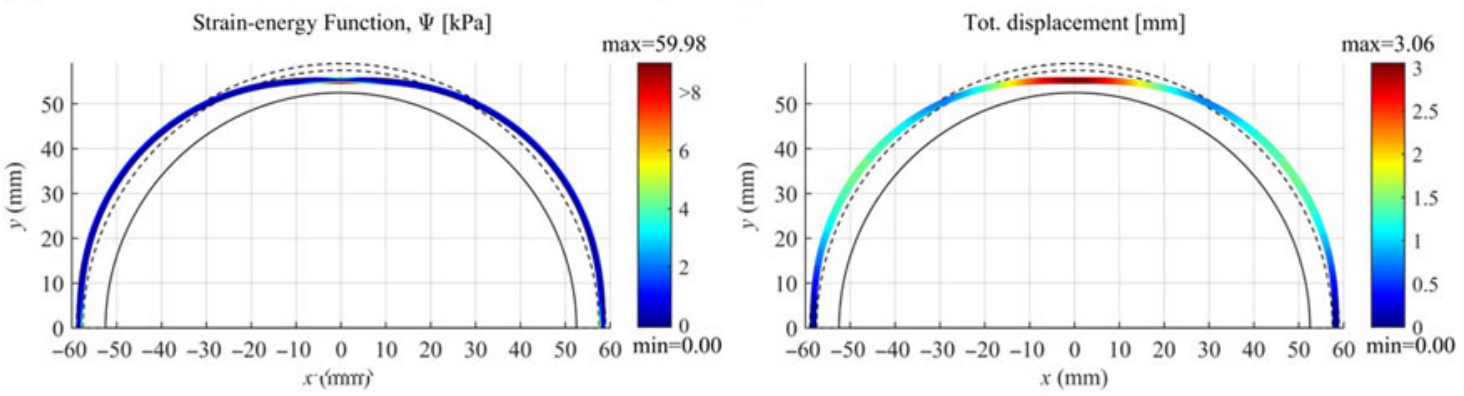

(b)
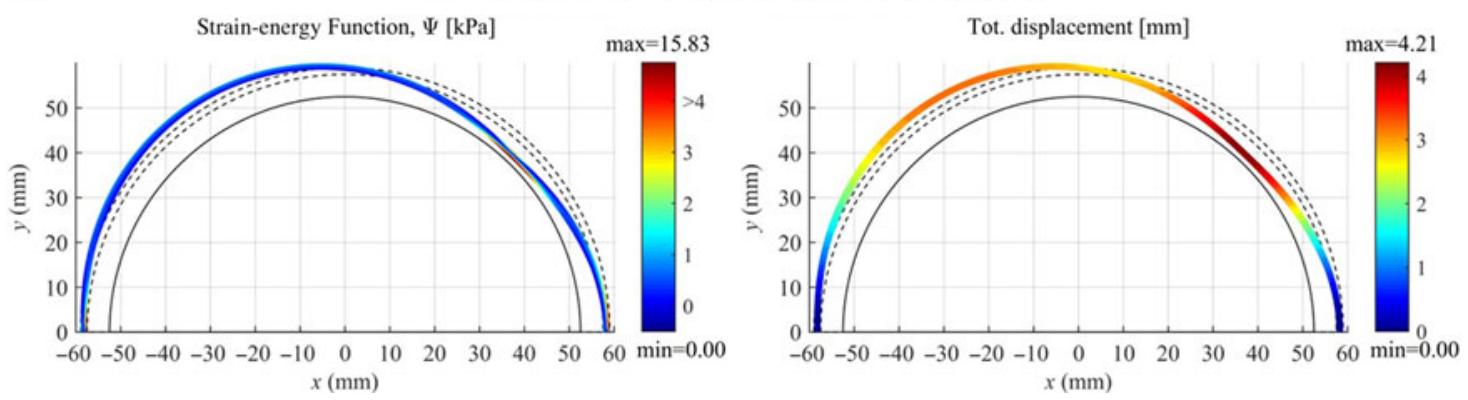

Fig. 18. Strain-energy function, $\Psi$, and local displacement of the membrane points. (a) Case A: concentrated central load. Plots refer to the equilibrium configuration with oil differential pressure of $10 \mathrm{kPa}$ and load of 24.4 $\mathrm{N}$ (uniformly distributed over the device width). (b) Case B: concentrated inclined load (at $45^{\circ}$ with respect to the axis). Plots refer to the equilibrium configuration with oil differential pressure of $10 \mathrm{kPa}$ and load of $20.7 \mathrm{~N}$ (uniformly distributed over the device width).

In Fig. 18, we report the results relative to a scenario in which the oil equilibrium pressure difference is $10 \mathrm{kPa}$. Figure 18(a) is relative to loading case A (central load, Fig. 17), while Fig. 18(b) is for case B (load at $45^{\circ}$, as shown in Fig. 17). In each sub-figure of Fig. 18, the plot on the left is a contour plot showing the strain-energy density, $\Psi$, of the material (which provides an energetic representation of the level of stress), and the contour plot on the right shows the module of the total displacement. In the graphs, the undeformed membrane configuration is represented with dashed lines, while the inner rigid wall of the oil chamber is in black solid line.

As expected with the oil volume conservation, the pattern of deformation is such that the inward deformation due to the external load is compensated by a peripheral outward expansion of the membrane, induced by increased oil pressure.

The relation between prescribed load (total load spread on the cylinder width) and the resulting maximum displacement of the membrane is shown in Fig. 19. Markers refer to different FE simulations, while the two sets of data refer to the different loading conditions (central and inclined load). The plot shows that the force rises more than linearly with increasing membrane displacement as a result of the device becoming stiffer at large deformations due to the presence of the incompressible fluid film. Moreover, the slope of the curve in the central loading scenario has larger slope, that is, the device opposes greater stiffness when loaded centrally (rather than laterally), as the two sides of the membrane symmetrically react to the external load through their deformation.

The proposed FE method is a useful tool that allows prediction of the membrane displacements (as well as the overall deformed shape) due to external loads, over a wide set of applied forces and deformations. Different types of loads (e.g., pressure loads, instead of forces) can be easily simulated using the same numeric scheme and the proposed modeling strategy.

\section{Conclusion and Future Works}

In this paper a novel SUAS is proposed and realized using a 3D printer. Our proposed SUAS can be used as a distributed force sensor or as a contact detection device. It functions as a capacitive sensor and it features a hyperelastic membrane end effector, a conductive compliant electrode, a dielectric fluid film, and a layer integrating dielectric taxels and lower electrode. 


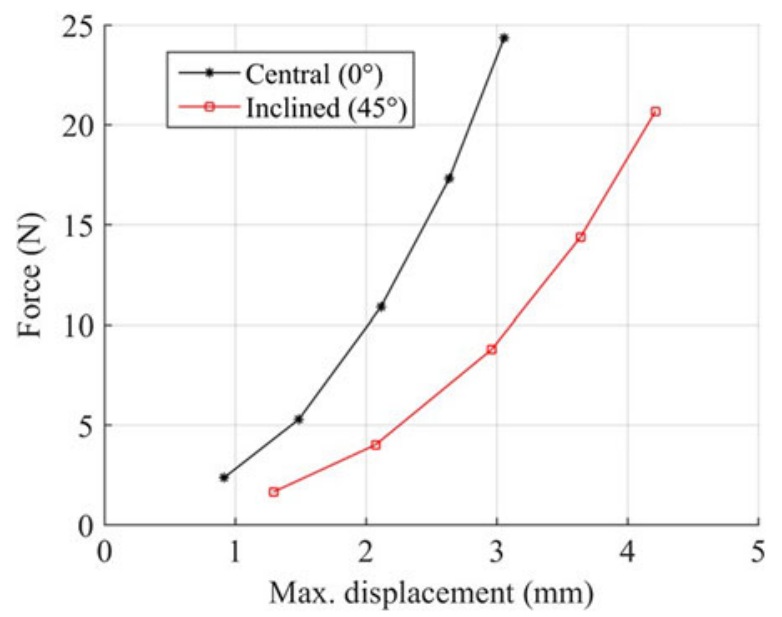

Fig. 19. Maximum membrane displacement as a function of the module of the concentrated load for the two scenarios (case A and case B).

In the paper, simulation on hyperelastic materials and experiments on conductive layers in oil have been presented. Simulation on hyperelastic membranes confirms EPDM as a good material for these types of applications.

When an external load is applied to the surface of our SUAS, the distance between conductive layer and transducers reduces at the point of contact, resulting in higher capacitance. If several loads are applied on the surface, the sensor recognizes higher capacitance points mapping the contact.

The results show low differences using PCBs in air or oil ambient. If the conductive layer is very compliant, the oil allows adhesion to the conductive layer with taxels giving a higher value of the capacitance with respect to the conductive layer without the soft layer. In conclusion, superior performance is obtained using conductive layers made of deformable tissue as used in the presented tests.

The repeatability and stability of the proposed SUAS are based on two points: (1) the evolution of the ROBOSKIN tactile sensor, where the repeatability is documented in many papers and in this paper the behavior of the triangular PCBs in oil and air shows a very interesting optimization and (2) the hyperelastic membrane which underlines how the elasticity of the used material allows to reproduce the offset conditions.

In order to avoid creeps on the soft membrane interacting with the fauna and flora of the deep-sea environment, future works are oriented to design a curved keyboard substituting the external rigid part of the coverage of the SUAS. A study on fluid-membrane interaction modifying the geometry of the sensor is planned for the future.

\section{Acknowledgments}

This paper is an extension of the work presented in the international conference OCEANS 2015. ${ }^{21}$ The research was conducted at the MACLAB (Mechatronics and Automatic Control Laboratory), at Polytechnic School of the University of Genova, and was partly supported by MIUR through the MARIS prot. 2010FBLHRJ project, supporting the GRAAL Laboratory and by the European Commission in the FP7/2007-2013 ROBOSKIN Project (Contract No. 231500) supporting the MACLAB.

\section{References}

1. http://ec.europa.eu/maritimeaffairs/policy/blue_growth/index_en.htm 12/02/2016.

2. R. Bogue, "Underwater robots: A review of technologies and applications," Ind. Rob. Int. J. 42(3), 186-191 (2015).

3. J. R. Bemfica, C. Melchiorri, L. Moriello, G. Palli and U. Scarcia, "A Three-Fingered Cable-Driven Gripper for Underwater Applications," 2014 IEEE International Conference on Robotics and Automation (ICRA), Hong Kong (2014) pp. 2469-2474.

4. T. Ding-Zhong, W. Qi-Ming, S. Rui-Han, Y. Xin and G. Yi-Hua, "Optical fiber based tactile sensor for underwater robots," J. Marine Sci. Appl. 7, 122-126 (2008). doi:10.1007/s11804-008-7055-3. 
5. D. M. Lane, J. B. C. Davies, G. Robinson, D. J. O’Brien, J. Sneddon, E. Seaton and A. Elfstrom, “The AMADEUS dextrous subsea hand: Design, modeling, and sensor processing," IEEE J. Oceanic Eng. 24(1), 96-111 (1999).

6. P. Liljebäck, Ø. Stavdahl, K. Y. Pettersen and J. T. Gravdahl, "Mamba-A waterproof snake robot with tactile sensing," 2014 IEEE/RSJ International Conference on Intelligent Robots and Systems (IROS 2014), Chicago (IEEE, September 14-18, 2014) pp. 294-301.

7. X. DeZhang, Y. J. Ge, S. Fei, W. Z. Cheng, G. L. Fu and N. Yuman, "Tactile Sensing for Underwater Operation System Based on Multi Finger Sensors Information Fusion," 2005 IEEE International Conference on Information Acquisition, Hong Kong and Macau (IEEE, 2005) p. 6.

8. J. Lemburg, P. Kampmann and F. Kirchner, "A Small-Scale Actuator with Passive-Compliance for a FineManipulation Deep-Sea Manipulator," OCEANS 2011, Kona, Hawaii (IEEE, 2011) pp. 1-4.

9. G. Palli, L. Moriello and C. Melchiorri, "Performance and sealing material evaluation in 6-axis force-torque sensors for underwater robotics," IFAC-PapersOnLine 48(2), 177-182 (2015).

10. H. S. Stuart, M. Bagheri, S. Wang, H. Barnard, A. L. Sheng, M. Jenkins and M. R. Cutkosky, "Suction Helps in a Pinch: Improving Underwater Manipulation with Gentle Suction Flow," 2015 IEEE/RSJ International Conference on Intelligent Robots and Systems (IROS), Hamburg, Germany (IEEE, 2015) pp. 2279-2284.

11. H. S. Stuart, S. Wang, O. Khatib and M. R. Cutkosky, "The ocean one hands: An adaptive design for robust marine manipulation," Int. J. Rob. Res. 36(2), 150-166 (2017). doi:10.1177/0278364917694723.

12. A. Aggarwal, P. Kampmann, J. Lemburg and F. Kirchner, "Haptic object recognition in underwater and deep-sea environments," J. Field Rob. 32, 1-19 (2014). doi:10.1002/rob.21538.

13. C. M. Oddo, L. Beccai, G. G. Muscolo and M. C. Carrozza, "A Biomimetic MEMS-Based Tactile Sensor Array with Fingerprints Integrated in a Robotic Fingertip for Artificial Roughness Encoding," Proceedings IEEE 2009 International Conference on Robotics and Biomimetics (ROBIO 2009), Guilin, Guangxi, China (2009).

14. D. Silvera-Tawil, D. Rye and M. Velonaki, "Artificial skin and tactile sensing for socially interactive robots: A review," Rob. Auton. Syst. 63, 230-243 (2015).

15. A. Bruno and G. Cannata, "A New Tactile Sensor for Robotic Underwater Applications," IIA/SOCO, Genova, Italy (1999).

16. M. Asadnia, A. G. P. Kottapalli, J. Miao, M. E. Warkiani and M. S. Triantafyllou, "Artificial fish skin of self-powered micro-electromechanical systems hair cells for sensing hydrodynamic flow phenomena," J. R. Soc. Interface 12(111), 20150322 (2015).

17. P. R. Bandyopadhyay and A. M. Hellum, "Modeling how shark and dolphin skin patterns control transitional wall-turbulence vorticity patterns using spatiotemporal phase reset mechanisms," Sci. Rep. 4, 6650 (2014).

18. L. Wen, J. C. Weaver and G. V. Lauder, "Biomimetic shark skin: Design, fabrication and Hydrodynamic function," J. Exp. Biol. 217, 1656-1666 (2014). doi:10.1242/jeb.097097.

19. C. Walker and I. Anderson, "From Land to Water: Bringing Dielectric Elastomer Sensing to the Underwater Realm," In: Electroactive Polymer Actuators and Devices (EAPAD) 2016, vol. 9798, Las Vegas, NV (International Society for Optics and Photonics, 2016) p. 97982B.

20. J. C. Kahn and J. L. Tangorra, "The effects of fluidic loading on underwater contact sensing with robotic fins and beams," IEEE Trans. Haptic 9(2), 184-195 (2016).

21. G. G. Muscolo and G. Cannata, "A Novel Tactile Sensor for Underwater Applications: Limits and Perspectives," OCEANS 2015, Genova (IEEE, 2015) pp. 1-7.

22. G. Antonelli, Underwater Robots. Motion and Force Control of Vehicle-Manipulator Systems. Springer Tracts in Advanced Robotics (Springer-Verlag, Heidelberg, Germany, 2003).

23. J. Rajruangrabin and D. O. Popa, "Enhancement of Manipulator Interactivity Through Compliant Skin and Extended Kalman Filtering," IEEE International Conference on Automation Science and Engineering, 2007, CASE 2007 (IEEE, 2007) pp. 1111-1116.

24. A. Cirillo, P. Cirillo, G. De Maria, C. Natale and S. Pirozzi, "An artificial skin based on optoelectronic technology," Sens. Actuators, A 212, 110-122 (2014).

25. P. Mittendorfer, E. Dean and G. Cheng, "Automatic Robot Kinematic Modeling with a Modular Artificial Skin," 2014 14th IEEE-RAS International Conference on Humanoid Robots (Humanoids) (IEEE, 2014) pp. 749-754.

26. A. Schmitz, P. Maiolino, M. Maggiali, L. Natale, G. Cannata and G. Metta, "Methods and technologies for the implementation of large-scale robot tactile sensors," IEEE Trans. Rob. 27(3), 389-400 (2011).

27. D. Roy, N. Wettels and G. E. Loeb, "Elastomeric skin selection for a fluid-filled artificial fingertip," J. Appl. Polym. Sci. 127(6), 4624-4633 (2013).

28. P. Kampmann, J. Lemburg, H. Hanff and F. Kirchner, "Hybrid Pressure-Tolerant Electronics," IEEE OCEANS (2012).

29. C. Thiede, M. Buscher, M. Lück, H. Lehr, G. Körner, J. Martin, M. Schlichting, S. Krüger and H. Huth, "An Overall Pressure Tolerant Underwater Vehicle: DNS Pegel," OCEANS 2009-EUROPE, Bremen, Germany (2009) pp. 1-6.

30. R. Li, A. P. Anvar, A. M. Anvar and T.-F. Lu, "Dynamic modeling of underwater manipulator and its simulation," World Acad. Sci. Eng. Tehnol. 6, 2611-2620 (2012).

31. G. Casalino, G. Casalino, M. Caccia, S. Caselli, C. Melchiorri, G. Antonelli, A. Caiti, G. Indiveri, G. Cannata, E. Simetti, S. Torelli and A. Sperindè, "Underwater intervention robotics: An outline of the Italian national project Maris,” Mar. Technol. Soc. J. 50(4), 98-107 (2016). 
32. G. Cannata and M. Maggiali, "An Embedded Tactile and Force Sensor for Robotic Manipulation and Grasping," Proceedings of 2005 IEEE-RAS International Conference on Humanoid Robots, Tsukuba, Japan (2005).

33. P. Maiolino, M. Maggiali, G. Cannata, G. Metta and L. Natale, "A flexible and robust large scale capacitive tactile system for robots," IEEE Sens. J. 13(10), 3910-3917 (2013). doi:10.1109/JSEN.2013.2258149.

34. E. Simetti, G. Casalino, S. Torelli, A. Sperindè and A. Turetta, "Floating underwater manipulation: Developed control methodology and experimental validation within the TRIDENT project," J. Field Rob. 31(3), 364-385 (2014).

35. G. A. Holzapfel, Nonlinear Solid Mechanics. A Continuum Approach for Engineering (John Wiley \& Sons, Ltd., West Sussex, England, 2000).

36. R. W. Ogden, "Elastic Deformations of Rubberlike Solids," In: Mechanics of Solids, the Rodney Hill 60th Anniversary Volume (H. G. Hopkins and M. J. Sewell, eds.), (Pergamon Press, Oxford, 1982) pp. 499-537.

37. L. Dorfmann and R. W. Ogden, "Nonlinear theory of electrostatic and magnetoelastic interactions," In: Nonlinear Elasticity Background, Chapter 3 (Springer, 2014) pp. 83-86. doi:10.1007/ 978-1-4614-9596-3_3.

38. R. Abeyaratne, Lecture Notes on the Mechanics of Elastic Solids. Volume II: Continuum Mechanics, 1st edn (Cambridge, MA and Singapore, 1952).

39. D. Felhos, D. Xu, A. K. Schlarb, K. Váradi and T. Goda, "Viscoelastic characterization of an EPDM rubber and finite element simulation of its dry rolling friction," Express Polym. Lett. 2(3), 157-164 (2008).

40. B. Song and W. Chen, "One-dimensional dynamic compressive behavior of EPDM rubber," J. Eng. Mater. Technol. 125(3), 294-301 (2003).

41. R. Vertechy, G. P. P. Rosati and M. Fontana, "Reduced model and application of inflating circular diaphragm dielectric elastomer generators for wave energy harvesting," J. Vib. Acoust. 137(1), 011004 (2015).

42. A. D. Marchese, R. K. Katzschmann and D. Rus, "A recipe for soft fluidic elastomer robots," Soft Rob. 2(1), 7-25 (2015).

43. http://salvimar.com (October 31, 2016).

44. J. S. Muraoka, Deep-Ocean Biodeterioration of Materials - Part III. Three Years at 5300 Feet NCEL TR-428 (U.S. Naval Civil Engineering Laboratory, 1966). 